\title{
CONTINUOUS EXPLICIT RUNGE-KUTTA METHODS OF ORDER 5
}

\author{
J. H. VERNER AND M. ZENNARO
}

\begin{abstract}
A continuous explicit Runge-Kutta (CERK) method provides a continuous approximation to an initial value problem. Such a method may be obtained by appending additional stages to a discrete method, or alternatively by solving the appropriate order conditions directly. Owren and Zennaro have shown for order 5 that the latter approach yields some CERK methods that require fewer derivative evaluations than methods obtained by appending stages. In contrast, continuous methods of order 6 that require the minimum number of stages can be obtained by appending additional stages to certain discrete methods. This article begins a study to understand why this occurs. By making no assumptions to simplify solution of the order conditions, the existence of other types of CERK methods of order 5 is established. While methods of the new families may not be as good for implementation as the Owren-Zennaro methods, the structure is expected to lead to a better understanding of how to construct families of methods of higher order.
\end{abstract}

\section{INTRODUCTION}

Matched pairs of discrete explicit Runge-Kutta methods such as those derived by Fehlberg [4], Verner [9], and Prince and Dormand [7] are efficient algorithms for treating initial value problems of ordinary differential equations. Recently, it has been shown how the approximate solution provided by an explicit Runge-Kutta method at a set of discrete points can be extended to yield a continuous, or even differentiable, approximation. A bootstrapping technique developed by Enright et al. [3] appends extra stages to some well-known pairs to yield formulas that provide interpolated values of the solution over the complete interval of integration. In an alternative general-purpose strategy for appending stages, Verner [9] solved the order conditions sequentially to obtain such efficient interpolants of higher orders.

In a different approach for deriving continuous Runge-Kutta methods, Owren and Zennaro [5] establish the minimum numbers of stages required for continuous methods of orders $p \leq 5$. Furthermore, in [6] these authors exploit the strategy of stage-reuse to obtain eight-stage continuous methods of order 5 in

Received by the editor August 23, 1993 and, in revised form, May 2, 1994.

1991 Mathematics Subject Classification. Primary 65L06.

Key words and phrases. Interpolants, continuous explicit Runge-Kutta methods.

The work of the first author was supported by the Natural Sciences and Engineering Research Council of Canada, the Information Technology Research Centre of Ontario, and Consiglio Nazionale delle Ricerche of Italy.

The work of the second author was supported by the Ministero dell'Università e della Ricerca Scientifica e Tecnologica of Italy. 
which the First Stage of a new step is the same As the Last stage (FSAL) of the immediately preceding step of computation. Thus, after each successful step only seven new derivative evaluations would be required for the next step. Such a method requires one more stage than the seven-stage discrete FSAL pair of orders 4 and 5 constructed by Dormand and Prince [2], and simultaneously provides a continuous approximation. Since each continuous fifth-order FSAL method properly imbeds a fourth-order method, such a method may be implemented with a variable stepsize for effective error control.

For pairs of methods of orders 4 and 5 with appended interpolants constructed by Enright et al. [3], nine stages were required, and for other pairs of these orders, at least nine stages are required for such interpolants. In recent work, Santo [8] established that at least eleven stages are required for CERK methods of order 6, and Verner [10] has shown that eleven-stage continuously differentiable methods of order 6 could be obtained by appending interpolants to eight-stage pairs of orders 5 and 6 . That is, although minimal-stage CERK methods of order 5 cannot be obtained by appending interpolants to minimalstage pairs of orders 4 and 5, appending suitable stages to a minimal-stage pair of orders 5 and 6 can yield a minimal-stage CERK method of order 6 .

To gain a better understanding of minimal-stage continuous methods, we modify the approach in [5] to characterize the order conditions in a form analogous to that in [10]. Thus, we begin with a discrete method of order $p$ whose coefficients satisfy the set of (primary) quadrature conditions on the weights $\left\{b_{i}\right\}$ and nodes $\left\{c_{i}\right\}$,

$$
\sum_{i=1}^{s} b_{i} c_{i}^{\tau-1}=\frac{1}{\tau}, \quad \tau=1, \ldots, p,
$$

and the set of (secondary) subquadrature conditions which require that certain weighted sums of the subquadrature expressions using coefficients $\left\{a_{i j}\right\}$,

$$
q_{i}^{\tau}=\sum_{j=1}^{i-1} a_{i j} c_{j}^{\tau-1}-\frac{c_{i}^{\tau}}{\tau}, \quad \tau=1, \ldots, p_{i}, \quad i=1, \ldots, s,
$$

be identically zero. Although they differ from the standard form, these order conditions retain the one-to-one correspondence with rooted trees identified by Butcher [1]. Furthermore, the two different sets of order conditions are equivalent in the sense that coefficients for a method of order $p$ will satisfy both sets.

Familiarity with the use of rooted trees in tabulating the standard order conditions is assumed. In particular, each quadrature condition (1) is identified by a rooted tree of height $H(t)=1$ or 2 . The other subquadrature conditions differ from those in [1], and each of these corresponds to a tree $t=\left[t^{\prime}\right] \equiv$ $\left[\hat{t}_{1}, \hat{t}_{2}, \ldots, \hat{t}_{k}\right]$ of height $H(t) \geq 3$ with $r(t)=1+r\left(\hat{t}_{1}\right)+r\left(\hat{t}_{2}\right)+\cdots+r\left(\hat{t}_{k}\right)$ nodes, where $t^{\prime}$ is the set of trees $\left\{\hat{t}_{j}, j=1, \ldots, k\right\}$ obtained by deleting the root of $t$. This order condition may be represented formally as

$$
\psi(t) \equiv \sum_{i=1}^{s} b_{i}\left(\prod_{j=1}^{k} \psi_{i}\left(\hat{t}_{j}\right)\right)=0, \quad H(t) \geq 3, \quad 3 \leq r(t) \leq p .
$$

For each tree $t$, the product $\prod_{j=1}^{k} \psi_{i}\left(\hat{t}_{j}\right)$ of compound weights for stage $i$ is characterized by subquadratures and coefficients of a method uniquely identified 
by the set of trees $t^{\prime}$. Later, these are tabulated explicitly for $p \leq 5$, and otherwise defined recursively for all $p$. To construct a continuous method, we need to choose coefficients of a Butcher tableau

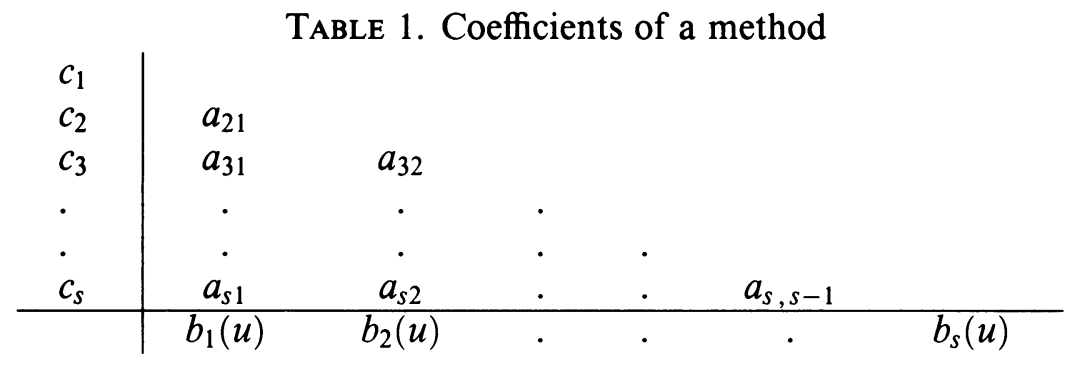

with polynomial weight functions $\left\{b_{i}(u), i=1, \ldots, s\right\}$ to satisfy the more general conditions

$$
\sum_{i=1}^{s} b_{i}(u) c_{i}^{\tau-1}=\frac{u^{\tau}}{\tau}, \quad \tau=1, \ldots, p
$$

and

$$
\psi(t)(u) \equiv \sum_{i=1}^{s} b_{i}(u)\left(\prod_{j=1}^{k} \psi_{i}\left(\hat{t}_{j}\right)\right)=0, \quad H(t) \geq 3, \quad 3 \leq r(t)<p .
$$

More detail on these order conditions is to be found in Verner [10].

By representing the tableau using an $s \times s$ matrix $A$, and $s$-vectors $\mathbf{b}(u)$ and c as

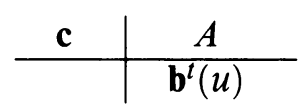

the order conditions may be written more concisely. Let e define the $s$-vector of units, $\mathbf{q}^{\tau}$ the $s$-vector of subquadratures of order $\tau$ whose elements are defined by (2), and $\mathrm{C}$ a diagonal matrix with diagonal $\mathbf{c}$. Then the quadrature conditions may be written as

$$
\mathbf{b}^{t}(u) C^{\tau-1} \mathbf{e}=\frac{u^{\tau}}{\tau} \equiv \int_{0}^{u} c^{\tau-1} d c, \quad \tau=1, \ldots, p .
$$

Observe that there exists a vector $\mathbf{b}(u)$ of weight functions satisfying these equations if and only if there are at least $p$ distinct nodes.

The subquadrature conditions may be written for order $3: \mathbf{b}^{t}(u) \mathbf{q}^{2}=0$, for order 4: $\quad \mathbf{b}^{t}(u) \mathbf{q}^{3}=\mathbf{b}^{t}(u) C \mathbf{q}^{2}=\mathbf{b}^{t}(u) A \mathbf{q}^{2}=0$, and for order 5: $\quad \mathbf{b}^{t}(u) \mathbf{q}^{4}=$ $\mathbf{b}^{t}(u) C \mathbf{q}^{3}=\mathbf{b}^{t}(u) C^{2} \mathbf{q}^{2}=\mathbf{b}^{t}(u) C A \mathbf{q}^{2}=\mathbf{b}^{t}(u) A \mathbf{q}^{3}=\mathbf{b}^{t}(u) A C \mathbf{q}^{2}=\mathbf{b}^{t}(u) A^{2} \mathbf{q}^{2}=$ $\mathbf{b}^{t}(u)\left(\mathbf{q}^{2}\right)^{2}=0$, where the square $\left(\mathbf{q}^{2}\right)^{2}$ of the vector $\mathbf{q}^{2}$ denotes a componentwise product. Thus, a continuous method of order 5 is obtained if the coefficients of $A$ and the vectors $\mathbf{c}$ and $\mathbf{b}(u)$ satisfy $\left(1^{\prime \prime}\right)$, and $\mathbf{b}^{t}(u)$ is orthogonal to twelve $s$-vectors. It is convenient to denote the matrix of these twelve column vectors as $\Psi_{5}^{\prime}$ with each column identified by the common degree of the homogeneous polynomials it contains. Hence, this matrix has one column of degree 2 , three columns of degree 3 , and eight columns of degree 4. 
For each order $p>5$, the subquadrature order conditions may be generated by constructing $\Psi_{r}^{\prime}, r=6, \ldots, p$, recursively. Then, the vector function $\mathbf{b}^{t}(u)$ must be orthogonal to the columns of $\Psi_{p}^{\prime}$. To obtain $\Psi_{r}^{\prime}$, columns of degree $r-1$ are appended to $\Psi_{r-1}^{\prime}$, possibly in the sequence corresponding to that suggested by Owren and Zennaro [5] for $\Phi_{r}$. However, unlike $\Phi_{r}$, $\Psi_{r}^{\prime}$ does not include the quadrature conditions and so the algorithm must be slightly modified. The first column of degree $r-1$ in $\Psi_{r}^{\prime}$ is $\mathbf{q}^{r-1}$. Next, $C$ transformations are obtained by premultiplying each column of degree $r-2$ of $\Psi_{r-1}^{\prime}$ by the diagonal matrix $C$. Then $A$-transformations are obtained by premultiplying each column of degree $r-2$ of $\Psi_{r-1}^{\prime}$ by $A$. Finally, each remaining column is the componentwise product of two columns of $\Psi_{r-3}^{\prime}$, the sum of whose degrees is exactly $r-1 \geq 4$.

For a continuous method of order $p$ characterized by a matrix $A$, Owren and Zennaro [5] define the matrix $A$ to be $p$-minimal if $\operatorname{dim}(A)=\operatorname{rank}\left(\Phi_{p}\right)$. Furthermore, they consider $\operatorname{CEN}(p)$, the minimum number of stages required for a CERK method of order $p$, and determine $\operatorname{CEN}(p)$ for $p \leq 5$. Here, it is convenient to define a CERK method of order $p$ to be $p$-minimal if its matrix $A$ is $p$-minimal, and $p$-optimal if it uses only $\operatorname{CEN}(p)$ stages. Results in [5] establish that some methods are not $p$-minimal, but that every $p$-optimal method is $p$-minimal. If $\Psi_{p}$ is obtained by appending $\Psi_{p}^{\prime}$ to the column matrix of $\left\{C^{\tau-1} \mathbf{e}, \tau=1, \ldots, p\right\}$, then it may be shown [10] that there is a nonsingular matrix $L$ for which $\Psi_{p}=L \Phi_{p}$, so that a method is $p$-minimal if $\operatorname{dim}(A)=\operatorname{rank}\left(\Psi_{p}\right)$. For example, this implies that the rows of $\Psi_{p}$ are linearly independent. Here, this representation of $p$-minimality is used to derive new $p$-optimal methods for $p=5$. To prove the results below and derive the new methods, a number of tedious algebraic computations are required, and these have all been executed using the MAPLE programming language. While the detailed computations are omitted, results of the computations are inserted as appropriate.

\section{Construction of MEthods}

We call the stage-order of stage $i$ the largest integer $p_{i}$ for which the subquadrature expressions (2) are equal to zero. That is, we shall assume for $\mathrm{SOV}=\left(p_{1}, \ldots, p_{s}\right)$, that

$$
q_{i}^{k}=0, \quad 1 \leq k \leq p_{i}, \quad i=1, \ldots, s,
$$

hold. This defines a stage-order vector (SOV) for each method, and we designate the dominant stage-order (DSO) as the least value of $p_{i} \in \mathrm{SOV}$ such that $\sum_{c_{1}=c_{1}} b_{j}(u) q_{j}^{p_{j}+1} \neq 0[10]$.

The 5-optimal CERK methods of the FSAL type constructed by Owren and Zennaro [6] have $\mathrm{DSO}=2$. An alternative derivation of those methods motivates the construction of other 5-optimal methods.

First, constrain the nodes to consist of subsets of distinct nodes so that the stage-order vector $(5,1,2,2,2,2,2,5)$ specifies the stage-orders of eight stages. The final stage has order 5 to allow for stage-reuse in the subsequent step.

Second, choose constants $K_{1}, K_{2}$, and $M_{r}, N_{r}, r=1,2,3$, to minimize 
the rank of $\Psi_{5}^{\prime}$. That is, for each $i=3, \ldots, 8$ impose the conditions

$$
q_{i}^{3} \equiv \sum_{j=1}^{i-1} a_{i j} c_{j}^{2}-\frac{c_{i}^{3}}{3}=K_{1} q_{i}^{2}+\sum_{k=2}^{8}\left\{K_{2} a_{i k}\right\} q_{k}^{2}
$$

(6) $\left(A^{2} \mathbf{q}^{2}\right)_{i} \equiv \sum_{k=2}^{8}\left\{\sum_{j=k+1}^{i-1} a_{i j} a_{j k} q_{k}^{2}\right\}=M_{1} q_{i}^{2}+\sum_{k=2}^{8}\left\{M_{2} a_{i k}+\left(c_{i}-c_{3}\right) M_{3} a_{i k}\right\} q_{k}^{2}$,

and

$$
q_{i}^{4} \equiv \sum_{j=1}^{i-1} a_{i j} c_{j}^{3}-\frac{c_{i}^{4}}{4}=N_{1} q_{i}^{2}+\sum_{k=2}^{8}\left\{N_{2} a_{i k}+\left(c_{i}-c_{3}\right) N_{3} a_{i k}\right\} q_{k}^{2} .
$$

Since $q_{k}^{2}=0$ if $k>2$, values of $K_{1}, M_{1}, N_{1}$ are of no consequence, and further, each summation over $k$ reduces to a mere substitution of $k=2$.

Third, for stage-reuse, stage $s=8$ must have stage-order 5 , so that $q_{8}^{\tau}=0$, $\tau=1, \ldots, 5$. Also, as $q_{k}^{2} \neq 0$ only for $k=2$, stage 2 also requires that

$$
a_{82}=\sum_{i=3}^{7} a_{8 i} a_{i 2}=\sum_{i=4}^{7} \sum_{j=3}^{i-1} a_{8 i} a_{i j} a_{j 2}=0 .
$$

When coefficients of a method are chosen to satisfy these constraints, the weight functions $\left\{b_{i}(u), i=1, \ldots, 8\right\}$ may be chosen to obtain a continuous method of order 5. This follows because the set $S_{C}=\left\{\mathbf{q}^{2}, A \mathbf{q}^{2}, C A \mathbf{q}^{2}\right\}$ is a basis for the column space of $\Psi_{5}^{\prime}$ : since $q_{k}^{2} \neq 0$ only if $k=2$, each of $C \mathbf{q}^{2}$, $C^{2} \mathbf{q}^{2}$, and $\left(\mathbf{q}^{2}\right)^{2}$ are multiples of $\mathbf{q}^{2}$, and also $A C \mathbf{q}^{2} \equiv c_{2} A \mathbf{q}^{2}$ is a multiple of $A \mathbf{q}^{2}$. Next, (5) implies that $\mathbf{q}^{3} \in \operatorname{span}\left\{\mathbf{q}^{2}, A \mathbf{q}^{2}\right\}$, and (6) and (7) imply that $A^{2} \mathbf{q}^{2}$ and $\mathbf{q}^{4} \in \operatorname{span}\left(S_{C}\right)$, respectively. Then, as $C \mathbf{q}^{2}, C A \mathbf{q}^{2}, A \mathbf{q}^{2}, A^{2} \mathbf{q}^{2} \in$ span $\left(S_{C}\right)$, (5) with premultiplication by each of $C$ and $A$ respectively implies that $C \mathbf{q}^{3}$ and $A \mathbf{q}^{3} \in \operatorname{span}\left(S_{C}\right)$. Hence, the twelve columns of $\Psi_{5}^{\prime}$ are spanned by $S_{C}$.

Thus, to obtain a continuous method of order 5, it is sufficient that the eight weight functions are chosen to satisfy $\left(1^{\prime \prime}\right)$ and are orthogonal to the set $S_{C}$. Such a choice is possible provided that the matrix of this system is nonsingular. Nonsingularity can be ensured by choosing certain nodes to be distinct and by imposing minor constraints on the coefficients.

It remains to show that the $s$ nodes and the $i-1$ coefficients of stage $i$ for each $i \leq 8$ can be selected to satisfy (4)-(8). To establish this, we observe first that the SOV imposes one condition on stage 2, two each on stages 3 to 7 , and five on stage 8 . Parameter $K_{2}$ is determined by stage 3 , and imposes one more condition on stage 4 . If the first four nodes are distinct, these uniquely determine coefficients of the first four stages. Parameters $\left\{M_{r}, N_{r}, r=2,3\right\}$ are then determined from stages 3 and 4 . The parameters are

$$
K_{2}=\frac{4 c_{3}-6 c_{2}}{3 c_{2}}
$$

$$
M_{2}=0, \quad M_{3}=\frac{c_{3}}{3 c_{3}-2 c_{4}}
$$


and

$$
N_{2}=\frac{c_{3}^{2}-2 c_{2}^{2}}{c_{2}}, \quad N_{3}=\frac{c_{3}\left(c_{4}-c_{3}\right)}{c_{2}\left(3 c_{3}-2 c_{4}\right)} .
$$

For stage 5 , the necessity for $\left\{a_{5 i}, i=1, \ldots, 4\right\}$ to satisfy (4) with $p_{5}=2$ and $(5)-(7)$ requires that the matrix

$$
F=\left(\begin{array}{ccccc}
1 & 1 & 1 & 1 & c_{5} \\
0 & c_{2} & c_{3} & c_{4} & \frac{c_{5}^{2}}{2} \\
0 & c_{2}^{2}-K_{2} q_{2}^{2} & c_{3}^{2} & c_{4}^{2} & \frac{c_{5}^{3}}{3} \\
0 & -M_{3}\left(c_{5}-c_{3}\right) q_{2}^{2} & a_{32} q_{2}^{2} & a_{42} q_{2}^{2} & 0 \\
0 & c_{2}^{3}-\left(N_{2}+N_{3}\left(c_{5}-c_{3}\right)\right) q_{2}^{2} & c_{3}^{3} & c_{4}^{3} & \frac{c_{5}^{4}}{4}
\end{array}\right)
$$

be singular. Since

$$
\operatorname{det}(F)=\frac{c_{2}^{2} c_{3}^{2} c_{4} c_{5}^{2}}{8\left(3 c_{3}-2 c_{4}\right)}\left(c_{3}-c_{4}\right)\left(c_{3}-c_{5}\right)\left(c_{4}-c_{5}\right)^{2},
$$

either one of $c_{2}, c_{3}, c_{4}, c_{5}$ is zero, or else two of the last three nodes are equal. If $c_{3}=c_{4}$, then $q_{4}^{2}=0$ and (5) for stage 4 would imply that $a_{43}=$ $c_{4}^{2}\left(c_{4}-c_{3}\right) / c_{3}^{2}=0$. Furthermore, $a_{42}=a_{32}$, so that stages 3 and 4 would be equivalent, and this would violate 5-minimality. If $c_{3}=c_{5}$, then $q_{3}^{2}=q_{5}^{2}$, and (5) and (7) for stages 3 and 5 lead to $a_{54} c_{4}\left(c_{3}-c_{4}\right) / 4=0$. If $a_{54}=0$, stages 3 and 5 would be equivalent. If $c_{4}=0$, then $a_{42}=a_{43}=0$, so that stages 1 and 4 would be equivalent, and either situation would violate 5 -minimality. Otherwise, $c_{3}=c_{4}$, which has just been precluded. Alternatively, choosing one of the nodes equal to zero also leads to equivalent stages, again violating 5minimality. Only the choice $c_{4}=c_{5}$ leads to an acceptable result. In this case, either $a_{54}=0$, which leads to the violation of 5-minimality, or else $c_{3}=c_{4}$ or $c_{3}=c_{4} / 2$. Only the latter of these is acceptable, and in this case, $F$ has rank 3 , and $a_{54}$ is arbitrary, but must be chosen nonzero.

The remaining stages are easily obtained. The five coefficients for stage 6 are uniquely determined by (4) with $p_{6}=2$ and (5)-(7) if $c_{2} \neq 0$, and $\left\{c_{1}=\right.$ $\left.0, c_{3}, c_{4}, c_{6}\right\}$ are distinct. For stage 7 , choose $a_{76}$ by equating the ratios of corresponding sides of the two equations

$$
a_{87} a_{76} c_{6}\left(c_{6}-c_{3}\right)\left(c_{6}-c_{4}\right)=\int_{0}^{1} \int_{0}^{c} c\left(c-c_{3}\right)\left(c-c_{4}\right) d c d \bar{c}
$$

and

$$
a_{87} c_{7}\left(c_{7}-c_{3}\right)\left(c_{7}-c_{4}\right)\left(c_{7}-c_{6}\right)=\int_{0}^{1} c\left(c-c_{3}\right)\left(c-c_{4}\right)\left(c-c_{6}\right) d c .
$$

(This requirement is sufficient with the following constraints on stage 8 to imply that $\sum_{i=3}^{7} a_{8 i} a_{i 2}=0$.) The remaining five coefficients for stage 7 are uniquely determined by (4) with $p_{7}=2$ and (5)-(7); the matrix is nonsingular because it is the same matrix as for stage 6 . The seven coefficients of stage 8 are constrained by (4) for $p_{8}=5$ and the annihilation of the first and third expressions in (8).

The choice of coefficients ensures that the vectors in $Q=\left\{\mathbf{e}, C \mathbf{e}, C^{2} \mathbf{e}\right.$, $C^{3} \mathbf{e}, C^{4} \mathbf{e}$ ) and $S_{C}$ are linearly independent. Hence, the weight functions can be chosen to satisfy $\left(1^{\prime \prime}\right)$, and to be orthogonal to $S_{C}$. Since $S_{C}$ spans $\Psi_{5}^{\prime}$, it 
follows that all of the order conditions are satisfied by the continuous method. Furthermore, this type of method admits stage-reuse. Observe that both stage 8 and the continuous method at the point $u=1$ are two stages of order 5 . Since the weights for these two stages are uniquely determined by the stages available for each, and eight independent order conditions are satisfied, it follows that the weights must be the same, and in particular, $b_{s}(1)=0$.

Let us now consider the construction of a more general CERK method of order $p$. We need to choose coefficients of $A$ and nodes of $\mathbf{c}$ so that, for the $s \times N_{p}$ matrix $\Psi_{p}$, there is a $p \times s$ solution $B_{p}$ to the matrix problem

$$
B_{p} \Psi_{p}=G_{p},
$$

where $G_{p}$ is the $p \times N_{p}$ matrix with $\left(G_{p}\right)_{i j}=\delta_{i j} / i$. For a $p$-minimal method, the rows of $\Psi_{p}$ will be linearly independent, $\Psi_{p}$ will have $p$ linearly independent quadrature columns, and the $N_{p}-p$ subquadrature columns remaining as $\Psi_{p}^{\prime}$ will form an $(s-p)$-dimensional subspace that will be orthogonal to the rows of the solution matrix $B_{p}$. In particular, $\mathbf{b}^{t}(u)=\left(u, u^{2}, \ldots, u^{p}\right) B_{p}$ is the vector of weight functions for such a continuous method. Only some $p$-minimal methods are $p$-optimal, and for $p=5$, some results are useful.

Lemma 1. For each 5-optimal CERK method, the following statements hold:

(a) the number of stages is $s=8$, and $\Psi_{5}^{\prime}$ has rank 3;

(b) $c_{2} \neq 0$;

(c) if $q_{3}^{2}=0$, then $c_{3} \neq 0$ and $a_{32} \neq 0$;

(d) if $q_{3}^{2}=q_{4}^{2}=0$, then either $S_{C}=\left\{\mathbf{q}^{2}, A \mathbf{q}^{2}, C A \mathbf{q}^{2}\right\}$ or $S_{A}=\left\{\mathbf{q}^{2}, A \mathbf{q}^{2}\right.$, $\left.A^{2} \mathbf{q}^{2}\right\}$ is a linearly independent set, and this independence occurs over stages 2,3 , and 4 ;

(e) at least six weight functions are nonzero.

Proof. (a) Owren and Zennaro [5] establish that at least eight stages are needed for a method of order 5, and, by construction, that eight-stage methods of order 5 exist. Observe that the five quadrature conditions of $\left(1^{\prime \prime}\right)$ imply that $\operatorname{rank}\left(B_{5}\right) \geq 5$, and so its nullspace, which contains the columns of $\Psi_{5}^{\prime}$, must have dimension not greater than 3 . If $\Psi_{5}^{\prime}$ has rank less than 3 , then it would be possible to solve all the order conditions of (11) with fewer than eight stages. Hence, the result follows.

(b) If $c_{2}=0$, stages 1 and 2 would be equivalent, and this would contradict 5-minimality. Hence, $c_{2} \neq 0$.

(c) If $c_{3}=0$, then $q_{3}^{2}=0$ implies that $a_{32}=0$, so that stages 1 and 3 are equivalent, and this would contradict 5-minimality. Hence, $c_{3} \neq 0$, and further, $q_{3}^{2}=0$ implies that $a_{32}=c_{3}^{2} /\left(2 c_{2}\right) \neq 0$.

(d) Assume that the vectors of $S_{C}$ are linearly dependent. Since $q_{1}^{2}=q_{3}^{2}=$ $q_{4}^{2}=0$ and $c_{3} a_{32} q_{2}^{2} \neq 0$, then every nonzero linear combination of $A q^{2}$ and $C A \mathbf{q}^{2}$ is linearly independent of $\mathbf{q}^{2}$. The linear dependence of $S_{C}$ then implies that $\left(C-c_{3} I\right) A \mathbf{q}^{2}=0$. Thus, either $c_{4}=c_{3}$ or $a_{42}=0$. Now suppose that $a_{43}=0$. Then $c_{4}=c_{3}$ and $q_{3}^{2}=q_{4}^{2}=0$ would imply the equivalence of stages 3 and 4 , a contradiction to 5-minimality. Otherwise, $a_{42}=0$ and $q_{4}^{2}=0$ would imply that stages 1 and 4 would be equivalent, again a contradiction to 5-minimality. Thus, $a_{43} \neq 0$, and so the form of $\mathbf{q}^{2}$ implies that $S_{A}$ is linearly independent. Hence, at least one of $S_{C}$ or $S_{A}$ is a linearly independent set and, as such, would be a basis of the column space of $\Psi_{5}^{\prime}$. 
(e) The arguments used to prove this result are intricate and may be of interest. A sketch follows, and a complete proof may be found in the Appendix of [11]. The quadrature conditions imply for any four nodes that

$$
\sum_{i=1}^{8} b_{i}^{\prime}(u) \prod_{k=1}^{4}\left(c_{i}-c_{i_{k}}\right)=\prod_{k=1}^{4}\left(u-c_{i_{k}}\right) \not \equiv 0,
$$

and this implies that at least five distinct nodes exist with nonzero weights. If there were precisely five nonzero weights in the vector $\mathbf{b}^{t}(u)$, and $\tilde{P}$ were the projection onto the support of $\mathbf{b}^{t}(u)$ (a subspace of $\mathbb{R}^{8}$ which is spanned by $\tilde{P} Q), \tilde{P}$ would annihilate the columns of $\Psi_{5}^{\prime}$. This would imply that $b_{i}(u) \neq 0$ only for $i=1,5,6,7,8$ and that

$$
\left[A\left(C-c_{5} I\right) \mathbf{q}^{2}\right]_{i}=\left[\mathbf{q}^{4}-\left(c_{2}+c_{5}\right) \mathbf{q}^{3}+c_{2} c_{5} \mathbf{q}^{2}\right]_{i}=0, \quad i=5,6,7,8 .
$$

The four conditions (13) for $i=5,6$ contradict $c_{2} \neq 0$ and the distinctness of the remaining nodes. Hence, at least six weights of $\mathbf{b}_{i}(u)$ must be nonzero.

For the 5-optimal CERK methods derived in [6], $S_{C}$ is a linearly independent set of columns of $\Psi_{5}^{\prime}$, and is necessarily a basis for its column space. Hence, it follows that the linear dependence expressed by $(5)-(7)$ is necessary for the CERK methods of this design.

To construct more general methods, it would be helpful if a basis for the column space of $\Psi_{p}^{\prime}$ could be constructed generically, and the next result moves in this direction.

Proposition 1. For any CERK method of order $p$ with $\mathrm{DSO}=d, S_{d}^{p-d-1}=$ $\left\{\mathbf{q}^{d+1}, C \mathbf{q}^{d+1}, \ldots, C^{p-d-2} \mathbf{q}^{d+1}\right\}$ is a linearly independent set of columns of $\Psi_{p}^{\prime}$.

Proof. Since the dominant stage-order DSO $=d$, there are one or more distinct nodes $c_{i}$ for which $\sum_{c_{j}=c_{i}} b_{j}(u) q_{j}^{d+1} \neq 0$. Suppose that $\left\{c_{i_{j}}, j=1, \ldots\right.$, $p-d-k\}$ are precisely the distinct nodes with this property ( $k<0$ is possible). Then if $k>0$, the order conditions imply that

$$
\sum_{i=1}^{s} b_{i}(u)\left(c_{i}-c_{i_{1}}\right) \cdots\left(c_{i}-c_{i_{p-d-k-1}}\right) q_{i}^{d+1}=0 .
$$

Exactly one term on the left side is nonzero which is impossible. Thus $k \leq 0$, and it follows necessarily that there are at least $(p-d)$ distinct nodes with $q_{i}^{d+1} \neq 0$. Since $S_{d}^{p-d-1}$ is a subset of $(p-d-1)$ columns of $\Psi_{p}^{\prime}$, the distinctness of $(p-d-1)$ nodes for which $q_{i}^{d+1} \neq 0$ is sufficient to establish that the vectors of $S_{d}^{p-d-1}$ are linearly independent.

For example if $c_{2} \neq 2 c_{3} / 3$, observe that (5) implies that $\mathbf{q}^{3}$ is a multiple of $A \mathbf{q}^{2}$ so that $\left\{\mathbf{q}^{2}\right\} \cup S_{2}^{2}$ could replace the basis $S_{C}$ in the previous formulation of 5-optimal CERK methods with DSO $=2$. For CERK methods of order 5 with $\mathrm{DSO}=1$, we obtain a more complete characterization.

Corollary. For each 5-optimal CERK method with DSO $=1, S_{1}^{3}=\left\{\mathbf{q}^{2}, C \mathbf{q}^{2}\right.$, $\left.C^{2} \mathbf{q}^{2}\right\}$ is a basis for the column space of $\Psi_{5}^{\prime}$. 
Proof. For such a method, $\Psi_{5}^{\prime}$ has rank 3, and contains the three vectors of $S_{1}^{3}$. By Proposition $1, S_{1}^{3}$ is a linearly independent set, and the result follows.

In general, if $S_{1}^{s-p}$ is a basis of the column space of $\Psi_{p}^{\prime}$, certain properties are satisfied.

Proposition 2. Suppose an s-stage continuous method of order $p$ exists for which $S_{1}^{s-p}=\left\{C^{j} \mathbf{q}^{2}, j=0,1, \ldots, s-p-1\right\}$ forms a basis of the column space of $\Psi_{p}^{\prime}$. Then,

(a) at least $s-p$ nodes corresponding to nonzero values of $q_{i}^{2}$ are distinct, and

(b) if $q_{i}^{2}=0$, stage $i$ has order at least $p-1$. Furthermore, if the method is p-minimal, then

(c) for $p \geq 5, q_{i}^{2}=0$ for at least $m$ indices of $i=2,3, \ldots, s$, where $m=\min (s-p-1,2 p-s)$, and

(d) for equal nodes, $c_{i}$ and $c_{j}$, exactly one of $q_{i}^{2}$ and $q_{j}^{2}$ is zero, and exactly one is nonzero. (Hence, multiple nodes can have multiplicity at most equal to 2.)

Proof. (a) If $k$ is the number of distinct nodes with $q_{i}^{2} \neq 0$, then there are at most $k$ linearly independent vectors in the set $S_{1}^{s-p}$. Since this set of $s-p$ vectors is a basis, $k \geq s-p$.

(b) If $q_{i}^{2}=0$, then because $S_{1}^{s-p}$ is a basis of the column space of $\Psi_{p}^{\prime}$, all entries of row $i$ are zero. Since $q_{i}^{1}=0$ as well, this implies that all conditions of orders from 1 to $p-1$ are valid for stage $i$.

(c) Suppose that $q_{i}^{2}=0$ for $k$ indices of $i=2, \ldots, s$. To show $k \geq m$, we use the fact that the number of linearly independent rows of a matrix must be no greater than the number of vectors that span its columns. Define the matrix $\bar{\Psi}_{p}$ as that obtained by deleting the first column of $\Psi_{p}$, and by replacing row $i$ by a row of zeros for each $i$ with $q_{i}^{2}=0$. Observe that $q_{1}^{2}=0$, trivially, and that the first row of $\Psi_{p}$ is $(1,0, \ldots, 0)$. Since $p$-minimality implies the rows of $\Psi_{p}$ are linearly independent, it follows that $\bar{\Psi}_{p}$ is an $s \times\left(N_{p}-1\right)$ matrix with $s-1-k$ linearly independent rows. Also, define

$$
\tilde{\mathbf{e}}= \begin{cases}1 & \text { if } q_{i}^{2} \neq 0, \\ 0 & \text { if } q_{i}^{2}=0 .\end{cases}
$$

The condition $\mathbf{b}^{t}(u)\left(\mathbf{q}^{2}\right)^{2}=0$ of order 5 implies that $\left(\mathbf{q}^{2}\right)^{2}$ is a column of $\Psi_{p}^{\prime}$, and hence that $\left(\mathbf{q}^{2}\right)^{2}=\left(\lambda_{1} I+\lambda_{2} C+\cdots+\lambda_{s-p} C^{s-p-1}\right) \mathbf{q}^{2}$ for some constants $\left\{\lambda_{j}, j=1, \ldots, s-p\right\}$, or equivalently that

$$
\mathbf{q}^{2}=\left(\lambda_{1} I+\lambda_{2} C+\cdots+\lambda_{s-p} C^{s-p-1}\right) \tilde{\mathbf{e}} .
$$

The first $p-1$ columns of $\bar{\Psi}_{p}$, which correspond to quadrature columns of $\Psi_{p}$, are precisely $\left\{C \tilde{\mathbf{e}}, \ldots, C^{p-1} \tilde{\mathbf{e}}\right\}$. As $S_{1}^{s-p}$ is a basis of the column space of $\Psi_{p}^{\prime},\left(15^{\prime}\right)$ may be used to show that all remaining columns of $\bar{\Psi}_{p}$ are spanned by the set $\left\{\tilde{\mathbf{e}}, C \tilde{\mathbf{e}}, \ldots, C^{2 s-2 p-2} \tilde{\mathbf{e}}\right\}$. Thus, $\bar{\Psi}_{p}$ has a column space spanned by the $r+1$ vectors $\left\{\tilde{\mathbf{e}}, C \tilde{\mathbf{e}}, \ldots, C^{r} \tilde{\mathbf{e}}\right\}, r=\max (p-1,2 s-2 p-2)$, and contains exactly $s-1-k$ linearly independent rows. Therefore, $s-1-k \leq r+1$, or equivalently, $k \geq s-2-r=\min (s-p-1,2 p-s)=m$. 
(d) If $c_{i}=c_{j}$ and $q_{i}^{2}=q_{j}^{2}=0$, then rows $i$ and $j$ of $\Psi_{p}$ are identical, and the method is reducible, leading to a contradiction to $p$-minimality. On the other hand, if both $q_{i}^{2} \neq 0$ and $q_{j}^{2} \neq 0$, then equation $\left(15^{\prime}\right)$ is nontrivial for each of components $i$ and $j$. Thence, equality of $c_{i}$ and $c_{j}$ implies that $q_{i}^{2}=q_{j}^{2}$. Consequently, rows $i$ and $j$ of $\Psi_{p}$ are the same. Again, this leads to a contradiction to $p$-minimality.

Corollary. Suppose for a 5-optimal CERK method, $S_{1}^{3}=\left\{\mathbf{q}^{2}, C \mathbf{q}^{2}, C^{2} \mathbf{q}^{2}\right\}$ is a linearly independent set. Then $\mathrm{DSO}=1$.

Proof. Since $S_{1}^{3} \subset \Psi_{5}^{\prime}$ and is linearly independent, it is a basis of the column space of $\Psi_{5}^{\prime}$. To establish that DSO $=1$, we need to show there is an $i$ for which $\sum_{c_{j}=c_{i}} b_{j}(u) q_{j}^{2} \neq 0$. Since $S_{1}^{3}$ is a linearly independent set, there are at least three distinct nodes for which $q_{i}^{2} \neq 0$. Now, for each pair of equal nodes $c_{i}, c_{j}$, Proposition 2(d) implies that exactly one of $q_{i}^{2}$ and $q_{j}^{2}$ is nonzero. Hence, if DSO $>1$, then for each $i, \sum_{c_{j}=c_{i}} b_{j}(u) q_{j}^{2}=0$, so that $b_{i}(u)=0$ whenever $q_{i}^{2} \neq 0$, and there are at least three indices for which this is true. However, this contradicts Lemma 1(e). Hence, it follows that DSO $=1$.

For the remainder of this section, we consider only the construction of various types of 5-optimal CERK methods. Each type of method can be characterized by selecting a basis for the column space of $\Psi_{5}^{\prime}$, and for each type there are various choices available. Each choice leads to a family of methods, or else to a contradiction to the existence of a family of a particular type. The arguments to preclude some types rely on the fact that two identical rows of $\Psi_{5}$ would violate 5-minimality.

The main result characterizes all 5-optimal CERK methods with DSO = 1. By the corollary to Proposition $1, S_{1}^{3}=\left\{\mathbf{q}^{2}, C \mathbf{q}^{2}, C^{2} \mathbf{q}^{2}\right\}$ is a basis for the column space of $\Psi_{5}^{\prime}$. For these methods, certain coefficients are nonzero. Lemma 1(b) implies that $c_{2} \neq 0$, and if $q_{3}^{2}=0$, Lemma 1 (c) implies that $a_{32} \neq 0$. It would then follow that $\left(A \mathbf{q}^{2}\right)_{3} \equiv a_{32} q_{2}^{2} \neq 0$ while the third entry of each basis vector is zero, a contradiction. Hence, $q_{3}^{2} \neq 0$. Now since both $q_{2}^{2}$ and $q_{3}^{2}$ are nonzero, Proposition $2(\mathrm{~d})$ implies that $c_{2} \neq c_{3}$ (although possibly $\left.a_{32}=0\right)$. Next, assume that $q_{4}^{2}=0$, so that each entry in the fourth row of $\Psi_{5}^{\prime}$ is zero. If $a_{43}=0$, then $a_{42} q_{2}^{2} \equiv\left(A q^{2}\right)_{4}=0$, implying that $a_{42}=0$. Thus, $0=q_{4}^{2}=-c_{4}^{2} / 2$ so that stages 1 and 4 would be identical, a contradiction. Hence $a_{43} \neq 0$, and in turn this implies that $\left(A\left(C-c_{2} I\right) \mathbf{q}^{2}\right)_{4} \equiv a_{43}\left(c_{3}-c_{2}\right) q_{3}^{2} \neq$ 0 , a contradiction to having all entries of row 4 in $\Psi_{5}^{\prime}$ equal to zero. Thus, $q_{4}^{2}$ cannot be zero. Hence, elements 2, 3, and 4 of $\mathbf{q}^{2}$ are nonzero. Furthermore, by virtue of Proposition $2(\mathrm{~d})$, the nodes $\left\{c_{2}, c_{3}, c_{4}\right\}$ are distinct.

Since the rows of $\Psi_{5}^{\prime}$ are spanned by $S_{1}^{3}$, there are constants $J_{r}, K_{r}, L_{r}$, $M_{r}, N_{r}, r=1,2,3$, for which

$$
\begin{gathered}
\left(A \mathbf{q}^{2}\right)_{i} \equiv \sum_{j=1}^{i-1} a_{i j} q_{j}^{2}=\left\{J_{1}+J_{2}\left(c_{i}-c_{2}\right)\right\} q_{i}^{2}, \\
q_{i}^{3} \equiv \sum_{j=1}^{i-1} a_{i j} c_{j}^{2}-\frac{c_{i}^{3}}{3}=\left\{K_{1}+K_{2}\left(c_{i}-c_{2}\right)\right\} q_{i}^{2},
\end{gathered}
$$




$$
\begin{gathered}
\left(q_{i}^{2}\right)^{2}=\left\{L_{1}+\left(c_{i}-c_{2}\right)\left[L_{2}+L_{3}\left(c_{i}-c_{3}\right)\right]\right\} q_{i}^{2}, \\
\left(A\left(C-c_{2} I\right) \mathbf{q}^{2}\right)_{i} \equiv \sum_{j=1}^{i-1} a_{i j}\left(c_{j}-c_{2}\right) q_{j}^{2} \\
=\left\{M_{1}+\left(c_{i}-c_{2}\right)\left[M_{2}+M_{3}\left(c_{i}-c_{3}\right)\right]\right\} q_{i}^{2}
\end{gathered}
$$

and

$$
q_{i}^{4} \equiv \sum_{j=1}^{i-1} a_{i j} c_{j}^{3}-\frac{c_{i}^{4}}{4}=\left\{N_{1}+\left(c_{i}-c_{2}\right)\left[N_{2}+N_{3}\left(c_{i}-c_{3}\right)\right]\right\} q_{i}^{2} .
$$

In (16) and (17), it is assumed implicitly that $J_{3}=K_{3}=0$, and this requires justification, which is based on the following result.

Lemma 2. If $S_{1}^{3}=\left\{\mathbf{q}^{2}, C \mathbf{q}^{2}, C^{2} \mathbf{q}^{2}\right\}$ forms a basis of the column space of $\Psi_{5}^{\prime \prime}$ and in addition $q_{5}^{2}=0$, then $c_{4}=c_{5}$.

Proof. Since $q_{5}^{2}=0$, all entries in row 5 of $\Psi_{5}^{\prime}$ are zero, and by Proposition 2(b) the first five stages of this method yield a discrete method of order 4 scaled for the endpoint $c_{5}$. Butcher's derivation of such methods [1, p.177] implies that this is possible if and only if $c_{4}=c_{5}$. (In consequence, it follows that $a_{54} a_{43} a_{32} c_{2}=c_{5}^{4} / 24 \neq 0$, so each of $a_{32}, a_{43}, a_{54}$ must be nonzero.)

Now suppose to the contrary, that $J_{3} \neq 0$, and consider the right side of (16) extended to include the term $J_{3}\left(c_{i}-c_{2}\right)\left(c_{i}-c_{3}\right) q_{i}^{2}$. Since $C A \mathbf{q}^{2}$ is also a column of $\Psi_{5}^{\prime}$, premultiplication by $C$ of the extended form of (16) would imply that $C^{3} \mathbf{q}^{2}$ is spanned by the basis $S_{1}^{3}$. Together with the basis vectors, this would imply that $V=\left(C-c_{2} I\right)\left(C-c_{3} I\right)\left(C-c_{4} I\right) \mathbf{q}^{2}$ would be spanned by $S_{1}^{3}$, or equivalently by the basis $\left\{\mathbf{q}^{2},\left(C-c_{2} I\right) \mathbf{q}^{2},\left(C-c_{2} I\right)\left(C-c_{3} I\right) \mathbf{q}^{2}\right\}$ which is obtained by a triangular, unit diagonal composition of $S_{1}^{3}$. Since each of $q_{2}^{2}, q_{3}^{2}, q_{4}^{2}$ is nonzero, and nodes $c_{2}, c_{3}, c_{4}$ are distinct, the representation of $V$ in terms of the latter basis may be obtained using components 2,3 , and 4 . This representation implies $V \equiv 0$, and this would imply that $q_{i}^{2}=0$ for each of $i=5,6,7,8$. (Even if $c_{i}=c_{j}$ for some $j=2,3,4$, we have $q_{i}^{2}=0$ by Proposition 2(d).) In turn, this is found to be possible only if $c_{2}=0, c_{6}=0$ or $c_{6}=c_{4}$, each of which would imply violation of 5-minimality. To establish this, observe that $q_{5}^{2}=0$ implies by Lemma 2 that $c_{4}=c_{5}$. When $q_{6}^{2}=0$ as well, $q_{6}^{3}=q_{6}^{4}=0$ by (17) and (20), respectively, and then these three conditions would imply that

$$
\begin{aligned}
& a_{62}=\frac{c_{6}^{2}\left(3 c_{6}^{2}-4\left(c_{3}+c_{4}\right) c_{6}+6 c_{3} c_{4}\right)}{12 c_{2}\left(c_{2}-c_{3}\right)\left(c_{2}-c_{4}\right)} \text { and } \\
& a_{63}=\frac{c_{6}^{2}\left(3 c_{6}^{2}-4\left(c_{2}+c_{4}\right) c_{6}+6 c_{2} c_{4}\right)}{12 c_{3}\left(c_{3}-c_{2}\right)\left(c_{3}-c_{4}\right)} .
\end{aligned}
$$

Also, as stage 5 has order $4,\left(A \mathbf{q}^{2}\right)_{5}=\left(A\left(\mathbf{q}^{3}-c_{2} \mathbf{q}^{2}\right)\right)_{5}=0$ can be used to compute $a_{32}$ so that $q_{3}^{2}=c_{2} c_{3}\left(2 c_{3}-c_{5}\right) /\left(c_{3}-2 c_{2}\right)$. Then other order-4 conditions on stage 6 imply that

$$
\begin{aligned}
0 & =\left(A\left(C-c_{4} I\right) \mathbf{q}^{2}\right)_{6} \equiv a_{62}\left(c_{2}-c_{4}\right) q_{2}^{2}+a_{63}\left(c_{3}-c_{4}\right) q_{3}^{2} \\
& =\frac{c_{2} c_{6}^{2}\left(c_{4}-c_{6}\right)^{2}}{4\left(c_{5}-2 c_{2}\right)}
\end{aligned}
$$


which yields the stated restriction on the nodes. To avoid the resulting contradiction, it follows that at most one of $q_{5}^{2}$ and $q_{6}^{2}$ can be zero, and consequently $J_{3}=0$. Furthermore, using the same argument on $C \mathbf{q}^{3}$, it may be shown that $K_{3}=0$.

It is also possible to establish that $a_{54} a_{43} a_{32} \neq 0$ even if $q_{5}^{2} \neq 0$. Observe by using stages 2 and 3 on (16), we obtain

$$
J_{1}=0, \quad J_{2}=\frac{-a_{32} c_{2}^{2}}{\left(c_{3}-c_{2}\right)\left(2 a_{32} c_{2}-c_{3}^{2}\right)},
$$

and on (17), we obtain

$$
K_{1}=\frac{2 c_{2}}{3}, \quad K_{2}=\frac{2\left(a_{32} c_{2}^{2}-c_{3}^{3}+c_{2} c_{3}^{2}\right)}{3\left(c_{3}-c_{2}\right)\left(2 a_{32} c_{2}-c_{3}^{2}\right)} .
$$

Now $\left(16^{\prime}\right)$ implies $a_{32} \neq 0$, or otherwise we would get $A \mathbf{q}^{2}=0$, a contradiction to the following result.

Lemma 3. For a 5-optimal CERK method with $\mathrm{DSO}=1$, one has $A \mathbf{q}^{2} \neq 0$.

Proof. Assume to the contrary that $A \mathbf{q}^{2}=0$. In particular $\left(A \mathbf{q}^{2}\right)_{3}=0$, and so $q_{2}^{2} \neq 0$ implies that $a_{32}=0$. Then, from $\left(17^{\prime}\right), K_{2}=2 / 3$, and in this case, (17) becomes $\mathbf{q}^{3}=2 C \mathbf{q}^{2} / 3$. This is identical to $A C \mathbf{c}-C^{3} \mathbf{e} / 3=C$ $\left(2 A \mathbf{c}-C^{2} \mathbf{e}\right) / 3$, which implies that $A C \mathbf{c}=2 C A \mathbf{c} / 3$. A different representation yields $A \mathbf{c}^{2}=-2\left(A \mathbf{q}^{2}\right)+2 A(A \mathbf{c})=2 A(A \mathbf{c})$ under the assumption. Since $A$ is strictly lower triangular, then if $(A \mathbf{c})_{j}=0, j=1, \ldots, i-1$, the latter expression implies that $(A C \mathbf{c})_{i}=0$. If in addition $c_{i} \neq 0$, then the former expression implies in turn that $(A \mathbf{c})_{i}=0$.

For the methods considered, $a_{32}=0$ implies that $(A \mathbf{c})_{j}=0, j=1,2,3$, and it follows, using the previous argument inductively, that $\left(A \mathrm{c}^{2}\right)_{j}=0, j=$ $1,2,3,4$, and then that $(A \mathbf{c})_{4}=0$ since $c_{2}, c_{3}, c_{4}$ are nonzero. Furthermore, if $c_{i} \neq 0, i=5,6,7$, then by induction it follows that $A \mathrm{c}^{2}=0$. However, this precludes satisfaction of the order- 4 condition, $\mathbf{b}^{t}(u) A \mathbf{c}^{2}=u^{4} / 4$ when $u \neq 0$, and hence is not possible.

Otherwise, if $c_{i}=0$ for some $i=5,6,7$, we consider (18). For $j=$ $2,3,4$, we have $q_{j}^{2}=(A \mathbf{c})_{j}-c_{j}^{2} / 2=-c_{j}^{2} / 2 \neq 0$. Since coefficients $L_{1}, L_{2}, L_{3}$ in (18) are determined by stages $2,3,4$, uniquely, it follows that in this case (18) becomes $\left(\mathbf{q}^{2}\right)^{2}=-C^{2} \mathbf{q}^{2} / 2$. Thus, if $c_{i}=0$ for some $i=5,6,7$, then $q_{i}^{2}=0$, and so this stage would be equivalent to stage 1 thus violating 5minimality. Hence, the original assumption is impossible, and so $A \mathbf{q}^{2} \neq 0$.

To show that $a_{43} \neq 0$, assume otherwise. Then (16) and (17) for stage $i=4$ give two expressions from which the product term in $a_{32} a_{42}$ may be eliminated. Then distinctness of the nodes $c_{2}, c_{3}, c_{4}$, and the hypothesis $a_{43}=0$ would imply that $a_{32} c_{4}^{2}=a_{42} c_{3}^{2}$. Since $a_{32} \neq 0$, substitution of this expression for $a_{42}$ into (16) for $i=4$ would imply that $q_{4}^{2}=0$, a contradiction to the remark preceding (16). Hence, $a_{43} \neq 0$.

To prove that $a_{54} \neq 0$, assume otherwise. Also, since $q_{i}^{2} \neq 0, i=2,3,4,5$, Proposition 2(d) implies that $c_{2}, c_{3}, c_{4}$ and $c_{5}$ are distinct. Recall that $c_{2} \neq$ 
0 . Also $c_{3} \neq 0$, or else (17) for $i=4,5$ give values for $a_{42}$ and $a_{52}$, respectively, and then (18) for $i=5$ implies that $a_{32}\left(c_{4}-c_{5}\right)\left(c_{2}-c_{5}\right)=0$ which is not possible. Then (16) and (17) for $i=4,5$ form four linear equations in $a_{42}, a_{43}, a_{52}$ and $a_{53}$ with coefficients in terms of the nodes and $a_{32}$. On solving these formally and substituting, we find that (18) for $i=5$ is valid only if

$$
c_{2}^{5} a_{32}^{3}\left(c_{2}-c_{5}\right)\left(c_{3}-c_{5}\right)\left(c_{4}-c_{5}\right)\left(2 a_{32} c_{2}+c_{2} c_{3}-c_{3}^{2}\right)^{2}=0,
$$

and this can hold only if the final factor is zero. Thus,

$$
a_{32}=\frac{c_{3}\left(c_{3}-c_{2}\right)}{2 c_{2}}
$$

and in this case, each of $a_{42}, a_{43}, a_{52}$ and $a_{53}$ can be evaluated by their formal expressions since $c_{3} \neq 0$.

This choice of $a_{32}$ and $\left(16^{\prime}\right)$ lead to $J_{2}=1 / 2$, and $L_{1}=-c_{2}^{2} / 2, L_{2}=$ $-c_{2} / 2, \quad L_{3}=0$, so that

$$
q_{i}^{2}=-c_{2} c_{i} / 2, \quad i=2,3,4,5 .
$$

Also $\left(18^{\prime}\right)$ holds trivially for $i=1$. Using $\left(18^{\prime}\right)$ and (16), we obtain $-c_{2}(A \mathrm{c})_{6} / 2$ $\equiv \sum_{i=1}^{5} a_{6 i}\left(-c_{2} c_{i} / 2\right)=\sum_{i=1}^{5} a_{6 i} q_{i}^{2}=\left(c_{6}-c_{2}\right) q_{6}^{2} / 2$. If we assume $q_{6}^{2} \equiv$ $(A c)_{6}-c_{6}^{2} / 2=0$, then this would imply that $c_{6}=0$, which would preclude $5-$ minimality. Hence, $q_{6}^{2} \neq 0$, so that $\left(18^{\prime}\right)$ holds for $i=6$ as well. This argument may be repeated to imply that $-c_{2}(A \mathbf{c})_{7} / 2=\left(c_{7}-c_{2}\right) q_{7}^{2} / 2$, and therefore that $q_{7}^{2} \neq 0$. However, this is impossible since Proposition 2(c) implies that $q_{i}^{2}=0$ for at least two stages with $i \geq 2$. Hence, we conclude that $a_{54} \neq 0$.

Theorem 1. (a) There are three six-parameter families of 5-optimal CERK methods with $\mathrm{DSO}=1$.

(b) There are two three-parameter subsets of such methods that admit stagereuse.

(c) For each 5-optimal CERK method with DSO $=1$, one has $q_{5}^{2}=0$.

Proof. Because the proofs are quite technical, only sketches of how to construct the methods are given. The complete detail of these proofs may be found in [11].

(a) Construct a five-stage explicit Runge-Kutta method of order 4 scaled to the endpoint $c_{5}$, so that $q_{i}^{2} \neq 0, i=2,3,4$. (See Case 1 with $c_{3} \neq 1 / 2$, [1, p.179].) Appropriate choices of the constants in (16)-(20) are sufficient to satisfy the continuous order conditions, if in addition,

$$
c_{6}=\frac{2 c_{2} c_{3} c_{4}}{2 c_{2} c_{4}+2 c_{3} c_{4}-2 c_{2} c_{3}-c_{4}^{2}} .
$$

With either one or both of $q_{7}^{2}$ and $q_{8}^{2}$ equal to zero, three families of methods in the six parameters $\left\{c_{2}, c_{3}, c_{4}, c_{7}, c_{8}, a_{82}\right\}$ are obtained.

(b) Of these methods, some are of the FSAL type. For these, it is necessary that $c_{8}=1, b_{8}=0$, and either

$$
c_{3}=\frac{c_{4}\left(2 c_{2}-c_{4}\right)\left(60 c_{4}^{2}-105 c_{4}+48\right)}{c_{2}\left(60 c_{4}^{3}+80 c_{4}^{2}-270 c_{4}+144\right)-c_{4}\left(120 c_{4}^{2}-210 c_{4}+96\right)},
$$


or

$$
\begin{aligned}
c_{3}= & \frac{-c_{4} c_{7}\left(2 c_{2}-c_{4}\right)\left(12-15\left(c_{4}+c_{7}\right)+20 c_{4} c_{7}\right)}{\left[c _ { 2 } \left(c_{7}^{2}\left(60 c_{4}^{2}-160 c_{4}+90\right)-c_{7}\left(60 c_{4}^{3}-40 c_{4}^{2}-90 c_{4}+72\right)\right.\right.} \\
& \left.\left.+c_{4}\left(40 c_{4}^{2}-60 c_{4}+24\right)\right)+2 c_{4} c_{7}\left(12-15\left(c_{4}+c_{7}\right)+20 c_{4} c_{7}\right)\right]
\end{aligned}
$$

Either choice yields a family of methods in the three parameters $\left\{c_{2}, c_{4}, c_{7}\right\}$.

(c) This final result is established by contradiction involving an intricate expression obtained for $a_{32}$ using MAPLE.

This result characterizes all 5-optimal CERK methods with $S_{1}^{3}$ as a basis of the column space of $\Psi_{5}^{\prime}$. Among those methods with $q_{7}^{2}=0$, it is not possible to choose one for which stage 7 is the propagating stage (again, see [11] for the proof).

In summary, the Corollary to Proposition 1, and Theorem 1, characterize all eight-stage order-5 continuous methods with $\mathrm{DSO}=1$. Some particular examples are displayed in the next section.

The rest of this section surveys other 5-optimal CERK methods of order 5 that exist and each will have DSO $\geq 2$. As might be expected from the treatment at the beginning of this section, the characterization of all other eight-stage continuous methods of order 5 is based on the results of Owren and Zennaro [5, $6]$.

Proposition 3. A 5-optimal CERK method with $\mathrm{DSO} \geq 2, \mathrm{SOV}=\left(5,1, p_{3}\right.$, $\left.p_{4}, \quad p_{5}, p_{6}, p_{7}, p_{8}\right)$ and $c_{j} \neq c_{2}, j>2$, must satisfy $p_{j} \geq 2,3 \leq j \leq 8$.

Proof. The detailed proof of this result may be found in the Appendix to [11].

This result helps to identify the nature of continuous methods with DSO = 2. To establish whether or not there exist 5-optimal methods with DSO $\geq 2$ having one or more nodes coincident with $c_{2}$ and at least one stage other than the second with stage-order 1 would be a more tedious version of the same argument, and remains as an open question.

Arguments developed at the beginning of this section establish that $2 c_{3}=$ $c_{4}=c_{5}$ is sufficient to imply the existence of 5-optimal continuous FSAL methods with $\mathrm{DSO}=2$ as derived by Owren and Zennaro [6]. That development can be extended easily to characterize related continuous methods [5] without stage-reuse. To show that this constraint on the nodes is necessary for both types of methods, more is required.

Proposition 4. All 5-optimal CERK methods for which $\mathrm{SOV}=\left(5,1, p_{3}, p_{4}, p_{5}\right.$, $\left.p_{6}, p_{7}, p_{8}\right)$ with $p_{i} \geq 2, i \geq 3$ must satisfy $2 c_{3}=c_{4}=c_{5}$.

Proof. First, we show that $S_{C}=\left\{\mathbf{q}^{2}, A \mathbf{q}^{2}, C A \mathbf{q}^{2}\right\}$ is a basis for the column space of $\Psi_{5}^{\prime}$. Suppose to the contrary that $S_{C}$ is a linearly dependent set. As $q_{2}^{2} \neq 0$, and by Lemma $1(\mathrm{c}), a_{32} \neq 0$, the triangularity of $A$ and the linear dependence imply that $\left(C-c_{3} I\right) A \mathbf{q}^{2}=0$. Then Lemma $1(\mathrm{~d})$ implies that $a_{43} \neq 0$, and that $S_{A}=\left\{\mathbf{q}^{2}, A \mathbf{q}^{2}, A^{2} \mathbf{q}^{2}\right\}$ is a basis of the column space of $\Psi_{5}^{\prime}$.

Now, each of $\mathbf{q}^{3}, C \mathbf{q}^{3}, A \mathbf{q}^{3}, C A \mathbf{q}^{2}, \mathbf{q}^{4}$ can be represented in terms of the basis $S_{A}$. For $\mathbf{q}^{3}$, this representation is 


$$
\mathbf{q}^{3}=J_{1} \mathbf{q}^{2}+J_{2} A \mathbf{q}^{2}+J_{3} A^{2} \mathbf{q}^{2},
$$

and with $\left(C-c_{3} I\right) A \mathbf{q}^{2}=0$, the first four stages yield $J_{1}=2 c_{2} / 3, J_{2}=$ $\left(4 c_{3}-6 c_{2}\right) /\left(3 c_{2}\right)$, and $J_{3}=4\left(c_{4}^{2}\left(c_{4}-c_{3}\right)-a_{43} c_{3}^{2}\right) /\left(3 a_{43} c_{3}^{2} c_{2}\right)$. Suppose that $J_{3}=0$, so that $a_{43}=c_{4}^{2}\left(c_{4}-c_{3}\right) / c_{3}^{2}$. Since $a_{43} \neq 0, c_{4} \neq c_{3}$. In turn, $\left(c_{4}-c_{3}\right) a_{42} q_{2}^{2}=0$ would imply that $a_{42}=0$, and in this case, $q_{4}^{2}=0$ would imply that $a_{43}=c_{4}^{2} /\left(2 c_{3}\right)$, which with the previous expression for $a_{43}$ would imply that $c_{4}=3 c_{3} / 2$. That is, if $J_{3}=0$, then $a_{42}=0, c_{4}=3 c_{3} / 2$ and $a_{43}=$ $9 c_{3} / 8$. Furthermore, the assumption implies for stage 5 that $\left(c_{5}-c_{3}\right) a_{52}=0$. If $a_{52}=0$, then the resulting representations of $q_{5}^{3}$ and $q_{5}^{4}$ imply that $c_{5}=0$, $c_{3}=0$ and $a_{53}=0$, or $c_{5}=3 c_{3} / 2$ and $a_{53}=9 c_{3} / 8$. The first two imply the equivalence of stages 1 and 5 , and the third leads to the equivalence of stages 4 and 5. Otherwise, if $c_{5}=c_{3}$, the representations of $q_{5}^{3}$ and $q_{5}^{4}$ imply that $a_{52}=c_{3}^{2} /\left(2 c_{2}\right)$ and $a_{53}=a_{54}=0$, so that stages 3 and 5 would be equivalent. Since each of these contradicts 5 -minimality, it follows that $J_{3} \neq 0$.

Next, using stages 2, 3,4 to represent $A \mathbf{q}^{3}$ in terms of $S_{A}$, we obtain

$$
A \mathbf{q}^{3}=J_{1} A \mathbf{q}^{2}+J_{2} A^{2} \mathbf{q}^{2},
$$

since the form of $\mathbf{q}^{2}$ implies its coefficient in this expansion is zero. On computing the corresponding representation of $C \mathbf{q}^{3}$, we find

$$
C \mathbf{q}^{3}=J_{1} c_{2} \mathbf{q}^{2}+J_{2} c_{3} A \mathbf{q}^{2}+J_{3} c_{4} A^{2} \mathbf{q}^{2}
$$

by replacing $J_{2} c_{3} A \mathbf{q}^{2}$ by $J_{2} C A \mathbf{q}^{2}$ after finding the second cpefficient. Then, substituting $(25)$ into the left side of $\left(25^{\prime \prime}\right)$ yields

$$
0=J_{1}\left(C-c_{2} I\right) \mathbf{q}^{2}+J_{2}\left(C-c_{3} I\right) A \mathbf{q}^{2}+J_{3}\left(C-c_{4} I\right) A^{2} \mathbf{q}^{2} \equiv J_{3}\left(C-c_{4} I\right) A^{2} \mathbf{q}^{2}
$$

with the final equality following from the form of $\mathbf{q}^{2}$ and $C A \mathbf{q}^{2}=c_{3} A \mathbf{q}^{2}$. Similarly, substituting $(25)$ into $\left(25^{\prime}\right)$ yields

$$
0=J_{3} A^{3} \mathbf{q}^{2} \text {. }
$$

Since $J_{3} \neq 0$, then for stage $5,\left(26^{\prime}\right)$ implies that $a_{54}=0$. Then (26) implies that $\left(c_{5}-c_{4}\right) a_{53}=0$. Also the assumption implies that $\left(c_{4}-c_{3}\right) a_{42}=0$ and $\left(c_{5}-c_{3}\right) a_{52}=0$. If $a_{52}=a_{53}=0$, then $q_{5}^{2}=0$ would imply $c_{5}=0$, and hence that stages 1 and 5 are equivalent. If $a_{53}=0$ and $c_{5}=c_{3}$, then $q_{5}^{2}=0$ would imply that stages 3 and 5 are equivalent. If $a_{52}=a_{42}=0$ and $c_{5}=c_{4}$, then $q_{5}^{2}=0$ would imply that stages 4 and 5 are equivalent. Since each of these contradicts 5-minimality, the only feasible choice remaining is $c_{3}=c_{4}=c_{5}$ and $a_{54}=0$.

In the latter case, the assumption implies that $\left(c_{6}-c_{3}\right) a_{62}=0$. If $c_{6}=c_{3}$, then $q_{6}^{2}=0$ can be used to obtain $a_{62}$ since $c_{2} \neq 0$. Since $a_{43} \neq 0$ and $a_{54}=0,\left(26^{\prime}\right)$ can be used to obtain $a_{64}=-a_{65} a_{53} / a_{43}$. These choices make conditions for stage 6 consistent. However, in order to satisfy $a_{72}=0, q_{7}^{2}=$ 0 , and $\left(26^{\prime}\right)$, it follows that $c_{7}=c_{3}$ is required if $c_{7} q_{7}^{3}$ and $q_{7}^{4}$ are to be represented by the basis $S_{A}$. However, this would preclude the distinctness of at least five nodes, a contradiction to satisfying the five quadrature conditions.

Otherwise, if $a_{62}=0$, then $q_{6}^{2}=0$ yields $a_{63}$, and $\left(26^{\prime}\right)$ for stage 6 yields $a_{64}$. Then the representation of $q_{6}^{3}$ implies either $c_{6}=0$ or $c_{6}=c_{3}$. If $c_{6}=0$ and $q_{7}^{2}=0$, the representations of $q_{7}^{3}, q_{7}^{3}$ and $q_{7}^{4}$ imply that $c_{7}=0$, 
precluding the distinctness of at least five nodes. Otherwise, if $c_{6}=c_{3}$ and $q_{7}^{2}=0$, the representations for $q_{7}^{3},\left(A \mathbf{q}^{3}\right)_{7}$ and $c_{7} q_{7}^{3}$ imply that $c_{3}=c_{7}$, which again precludes the distinctness of at least five nodes. Finally, since $a_{62}=0$ implies $c_{6}=0$ or $c_{6}=c_{3}$, the choice $c_{7}=c_{3}$ would preclude the distinctness of five nodes. We can now conclude that $\left(C-c_{3} I\right) A \mathbf{q}^{2} \neq 0$.

It follows that $S_{C}=\left\{\mathbf{q}^{2}, A \mathbf{q}^{2}, C A \mathbf{q}^{2}\right\}$ is a basis of the column space of $\Psi_{5}^{\prime}$. However, in the continuing proof it is more convenient to represent the vectors $\mathbf{q}^{3}, A \mathbf{q}^{3}, C \mathbf{q}^{3}, A^{2} \mathbf{q}^{2}$ and $\mathbf{q}^{4}$ in terms of the equivalent basis $S_{\widehat{C}}=\left\{\mathbf{q}^{2}, A \mathbf{q}^{2},\left(C-c_{3} I\right) A \mathbf{q}^{2}\right\}$. Then we shall show that $\hat{J}_{3}=0$ in the representation

$$
\mathbf{q}^{3}=\hat{J}_{1} \mathbf{q}^{2}+\hat{J}_{2} A \mathbf{q}^{2}+\hat{J}_{3}\left(C-c_{3} I\right) A \mathbf{q}^{2},
$$

in order to establish the stated restriction on the nodes.

By obtaining the representations of $A q^{3}$ and $C \mathbf{q}^{3}$ in terms of $S_{\widehat{C}}$ and combining them with (27), it may be shown that

$$
\hat{J}_{3} A\left(C-c_{3} I\right) A \mathbf{q}^{2}=0
$$

(using an argument similar to that used for $\left(26^{\prime}\right)$ ). If $\hat{J}_{3} \neq 0$, this implies that $a_{54}\left(c_{4}-c_{3}\right)=0$. If $c_{4}=c_{3}$, then the representation of $A^{2} \mathbf{q}^{2}$ by $S_{\widehat{C}}$ implies that $a_{43}=0$, which leads to the equivalence of stages 3 and 4 , a contradiction to 5-minimality. If $a_{54}=0$, since $c_{3} \neq 0$, we can solve $q_{5}^{2}=0$ for $a_{53}$. Then the representation of $q_{5}^{3}$ and $\left(A \mathbf{q}^{3}\right)_{5}$ give equations in terms of $a_{42}, a_{52}$ and the nodes. For these equations to be satisfied, one of four constraints is necessary: (i) $c_{3}=c_{5}$, (ii) $c_{4}=c_{5}$, (iii) $c_{2}=2 c_{3} / 3$, or else (iv) $a_{42}=c_{4}^{2} /\left(2 c_{2}\right)$, $a_{52}=c_{5}^{2} /\left(2 c_{2}\right)$, and either $c_{3}=c_{5}$ or $c_{4}=c_{5}$. Since the final alternative is accommodated by the first two, we need only consider the first three alternatives. First, if $c_{3}=c_{5}$, either $c_{3}=0$ or stages 3 and 5 are equivalent, both of which are contradictions. Second, if $c_{4}=c_{5}$, then the representation of $A^{2} \mathbf{q}^{2}$ implies $a_{42}=a_{52}$, implying the equivalence of stages 4 and 5 , a contradiction, or else $c_{4}=0$. For the latter case, $c_{4}=c_{5}=0$, and all conditions for stage 5 are consistent. We need to consider stages 6 and 7. The representations of $\left(A q^{3}\right)_{6}$ and $q_{6}^{4}$ require that either $c_{6}=0$ or $c_{6}=c_{3}$. Then either value for $c_{6}$ and the representations of $\left(A \mathbf{q}^{3}\right)_{7}$ and $q_{7}^{4}$ require that either $c_{7}=0$ or $c_{7}=c_{3}$. These constraints on the nodes imply that $c_{1}=c_{4}=c_{5}=0$, and that $c_{6}$ and $c_{7}$ are equal to either zero or to $c_{3}$. In any of these cases, there will be no more than four distinct nodes for the eight stages. Since at least five nodes must be distinct to satisfy the quadrature conditions, this is a contradiction, so that $c_{4} \neq c_{5}$.

Finally, if $c_{2}=2 c_{3} / 3$, combining the representations of $\mathbf{q}^{3}$ with each of those for $c_{5} q_{5}^{3},\left(A^{2} \mathbf{q}^{2}\right)_{5}$, and $q_{5}^{4}$, implies $c_{4}=c_{5}$, which has been eliminated, $c_{2}=2 c_{5} / 3$, in which case stages 3 and 5 are equivalent, or $a_{42} c_{5}^{2}=a_{52} c_{4}^{2}$, which leads to the equivalence of stages 1 with 4 or 5 . Since all of these possibilities preclude 5-minimality, it follows that $\hat{J}_{3}=0$ in (27).

Since $\hat{J}_{3}=0$, it follows that $(27)$ is identical to (5). The argument developed from (5) shows that the nodes must be restricted as stated in the theorem.

This result characterizes all of the continuous methods found by Owren and Zennaro. The remaining results examine the particular continuous methods with $\mathrm{DSO} \geq 3$. 
Proposition 5. There is no 5-optimal CERK method for which (4) is valid for the $\mathrm{SOV}=\left(5,1,2, p_{4}, p_{5}, p_{6}, p_{7}, p_{8}\right)$ with $p_{i} \geq 3$ when $i \geq 4$.

Proof. Suppose, to the contrary, that such a method exists. By Lemma 1, $\Psi_{5}^{\prime}$ has rank 3 , and $c_{2}, c_{3}$ and $a_{32}$ are nonzero. By the SOV, $\mathbf{q}^{2}$ is a multiple of $(0,1,0,0,0,0,0,0)$, and $\mathbf{q}^{3}$ is a multiple of $\left(0, q_{2}^{3} / q_{3}^{3}, 1,0,0,0,0,0\right)$, so that a basis for the column space of $\Psi_{5}^{\prime}$ can be selected to include $q^{2}$ and $\mathbf{q}^{3}$. Hence, $\mathbf{b}^{t}(u) \mathbf{q}^{2}=\mathbf{b}^{t}(u) \mathbf{q}^{3}=0$ implies that $b_{2}(u)=b_{3}(u)=0$, and so $\mathbf{b}(u)$ is orthogonal to each of $\mathbf{q}^{2}, C \mathbf{q}^{2}, C^{2} \mathbf{q}^{2},\left(\mathbf{q}^{2}\right)^{2}, \mathbf{q}^{3}, C \mathbf{q}^{3}$. Also, if $\mathbf{b}(u)$ is orthogonal to $A \mathbf{q}^{2}$, it is also orthogonal to $A C \mathbf{q}^{2}$. Hence, for the method to have order 5 , it is necessary and sufficient that the coefficients and weights be restricted so that $\mathbf{b}(u)$ satisfies $\left(1^{\prime}\right)$, and is orthogonal to the seven vectors $\mathbf{q}^{2}, \mathbf{q}^{3}, A \mathbf{q}^{2}, A \mathbf{q}^{3}, C A \mathbf{q}^{2}, A^{2} \mathbf{q}^{2}, \mathbf{q}^{4}$.

We now show that any possible choices for stages 4 and 5 lead to a contradiction. If $c_{4}=0$, then $q_{4}^{2} \equiv a_{42} c_{2}+a_{43} c_{3}=0$ and $q_{4}^{3} \equiv a_{42} c_{2}^{2}+a_{43} c_{3}^{2}=0$ imply either $a_{42}=a_{43}=0$, or else $c_{2}=c_{3}$ and $a_{42}=-a_{43} \neq 0$. If $a_{42}=a_{43}=0$, stages 1 and 4 would be equivalent, a contradiction to 5-minimality. Otherwise, if $c_{2}=c_{3}$ and $a_{42}=-a_{43} \neq 0$, we can show that $c_{5}=\cdots=c_{8}=0$, and this is impossible since at least five of the nodes must be distinct in order to satisfy the quadrature conditions $\left(1^{\prime}\right)$. To show that $c_{5}=\cdots=c_{8}=0$, observe first that $a_{42} \neq 0$ together with arguments above imply that $\mathbf{q}^{2}, \mathbf{q}^{3}, A \mathbf{q}^{2}$ is a basis for the column space of $\Psi_{5}^{\prime}$. Furthermore, $c_{2}=c_{3}$ and $c_{4}=0$ imply that $q_{4}^{4}=0$. Hence $\mathbf{q}^{4}$ is spanned by the basis, and the first four stages imply that $\mathbf{q}^{4}=\left(-c_{2}^{2} / 2\right) \mathbf{q}^{2}+\left(3 c_{2} / 2\right) \mathbf{q}^{3}$. Since $p_{i} \geq 3$ for $i>3$, one has $q_{i}^{2}=q_{i}^{3}=0$ for $i>3$, and this implies that $q_{i}^{4}=0$ as well. Now assume that $c_{4}=\cdots=c_{k-1}=0$. Then $q_{k}^{2}=q_{k}^{3}=q_{k}^{4}=0$ imply that $a_{k 2} c_{2}^{\tau-1}+a_{k 3} c_{3}^{\tau-1}=c_{k}^{\tau} / \tau \equiv \int_{0}^{c_{k}} c^{\tau-1} d c$ for $\tau=2,3,4$. Since $c_{2}=c_{3}$, this in turn implies that $\int_{0}^{c_{k}} c\left(c-c_{2}\right)^{2} d c=0$, which is possible if and only if $c_{k}=0$. Hence, the equivalence of remaining nodes to zero follows by induction.

Alternatively, if $c_{4} \neq 0$, suppose that $a_{42}=0$. Then $q_{4}^{2}=q_{4}^{3}=0$ implies that $c_{4}=3 c_{3} / 2$, and $a_{43}=3 c_{4} / 4$. Furthermore, $q_{4}^{4}=-9 c_{3}^{4} / 64 \neq 0$, and so $\left\{\mathbf{q}^{2}, \mathbf{q}^{3}, \mathbf{q}^{4}\right\}$ forms a basis of the column space of $\Psi_{5}^{\prime}$. The first four stages are determined, and these are sufficient to characterize the representations of $A \mathbf{q}^{2}, A \mathbf{q}^{3}, C A \mathbf{q}^{2}, A^{2} \mathbf{q}^{2}$ in terms of the basis. Now, the coefficients of stage 5 are determined by $q_{5}^{2}=q_{5}^{3}=0$, and the representation of $\left(A q^{2}\right)_{5}$. Substitution of these coefficients into the representations of $\left(A \mathbf{q}^{2}\right)_{5}$ and $\left(A^{2} \mathbf{q}^{2}\right)_{5}$ admit only two choices for nodes $c_{3}$ and $c_{5}$. The first, $c_{3}=c_{5}=0$ leads to the equivalence of stages 5 and 1 , a contradiction to 5-minimality. The other requires that $c_{5}=3 c_{3} / 2=c_{4}, a_{54}=0$, and that stages 4 and 5 are equivalent, again contradicting 5-minimality.

It follows that $c_{4}$ and $a_{42}$ are nonzero. Thus, $\left\{\mathbf{q}^{2}, \mathbf{q}^{3}, A \mathbf{q}^{2}\right\}$ is a basis of the column space of $\Psi_{5}^{\prime}$. In this case, the coefficients $a_{5 i}, i=2,3,4$, are determined by $q_{5}^{2}=q_{5}^{3}=0$ and the representation of $q_{5}^{4}$ in terms of the basis. Then the corresponding representations of $A \mathbf{q}^{3}, C A \mathbf{q}^{2}$ and $A^{2} \mathbf{q}^{2}$ are possible only if $c_{5}=0, c_{4}=c_{5}$ or $c_{3}=3 c_{2} / 2$. If $c_{5}=0$, stages 5 and 1 are equivalent, and this violates 5-minimality; if $c_{4}=c_{5}$, stages 4 and 5 are equivalent, again violating 5-minimality. Finally, if $c_{3}=3 c_{2} / 2$, then $p_{3} \geq 3$, 
and this contradicts the specified value in the SOV. This establishes that no method of this type exists.

Proposition 6. Suppose that the nodes and coefficients of an eight-stage CERK method satisfy (4) for the $\mathrm{SOV}=\left(5,1,3, p_{4}, p_{5}, p_{6}, p_{7}, p_{8}\right)$ with $p_{i} \geq$ $3, i \geq 4$. Then this method has order 5 if and only if the coefficients are determined by (6) and (7), and the weights are determined by $\left(1^{\prime}\right)$.

Proof. Lemma 1 establishes that each of $c_{2}, c_{3}$, and $a_{32}$ is nonzero. The form of the SOV implies that both $\mathbf{q}^{2}$ and $\mathbf{q}^{3}$ are multiples of $(0,1,0,0,0,0,0,0)$ and are nonzero since $c_{2} \neq 0$. Hence, $\mathbf{b}^{t}(u) \mathbf{q}^{2}=0$ implies that $b_{2}(u)=0$, and so the vector $\mathbf{b}(u)$ is orthogonal to each of $\mathbf{q}^{2}, C \mathbf{q}^{2}, C^{2} \mathbf{q}^{2},\left(\mathbf{q}^{2}\right)^{2}, \mathbf{q}^{3}, C \mathbf{q}^{3}$. Also, if $\mathbf{b}(u)$ is orthogonal to $A \mathbf{q}^{2}$, it is also orthogonal to $A C \mathbf{q}^{2}$ and $A \mathbf{q}^{3}$. Hence, for the method to have order 5 , it is necessary and sufficient that the coefficients and weights be restricted so that $\mathbf{b}(u)$ satisfies $\left(1^{\prime}\right)$, and is orthogonal to the five vectors $\mathbf{q}^{2}, A \mathbf{q}^{2}, C A \mathbf{q}^{2}, A^{2} \mathbf{q}^{2}, \mathbf{q}^{4}$. The five linearly independent quadrature conditions imply that the column space of $\Psi_{5}^{\prime}$, which is the nullspace of $\mathbf{b}(u)$, has dimension three again. Next, we show that the three vectors $\mathbf{q}^{2}, A \mathbf{q}^{2}, C A \mathbf{q}^{2}$ are linearly independent so that this set forms a basis of the column space of $\Psi_{5}^{\prime}$.

As $q_{3}^{2}=0$ and entry 3 in each of $A \mathbf{q}^{2}$ and $C A \mathbf{q}^{2}$ is nonzero, these two vectors are linearly independent of $\mathbf{q}^{2}$. To show the two vectors are linearly independent of each other, assume otherwise, so that $\left(C-c_{3} I\right) A \mathbf{q}^{2}=0$. In particular, $\left(c_{4}-c_{3}\right) a_{42}=0$. However, $q_{3}^{2}=q_{3}^{3}=0$ implies that $c_{2}=2 c_{3} / 3$. Then if $c_{4}=c_{3}$ as well, $q_{3}^{2}=q_{3}^{3}=q_{4}^{2}=q_{4}^{3}=0$ would imply that $a_{43}=0$, and $a_{42}=a_{32}$, so that stages 3 and 4 would be equivalent, a contradiction to 5-minimality. Hence, $c_{4} \neq c_{3}$, and so $a_{42}=0$, implying in turn that $c_{3}=$ $2 c_{4} / 3$, and $a_{43}=3 c_{4} / 4 \neq 0$. Now, $\left(A^{2} \mathbf{q}^{2}\right)_{4} \equiv a_{43} a_{32} q_{2}^{2} \neq 0$, although $q_{4}^{2}$ and $\left(A \mathbf{q}^{2}\right)_{4} \equiv a_{42} q_{2}^{2}$ are both zero. This implies that the columns of $\Psi_{5}^{\prime}$ would be spanned by $\left\{\mathbf{q}^{2}, A \mathbf{q}^{2}, A^{2} \mathbf{q}^{2}\right\}$, and that $\mathbf{q}^{4}$ would be a linear combination of these vectors. Indeed, since the only remaining arbitrary choice for the first four stages would be $c_{2}$, a little computation yields $4 \mathbf{q}^{4}=\left(2 c_{2}^{2} I+c_{2} A+3 A^{2}\right) \mathbf{q}^{2}$. This condition together with $q_{5}^{2}=q_{5}^{3}=0$ determine the coefficients of stage 5. However, in this case $\left(c_{5}-c_{3}\right) a_{52}=0$ is also required. The choice $c_{5}=c_{3}$ with the computed coefficients of stage 5 would imply that stages 5 and 3 are equivalent, a contradiction to 5 -minimality. Otherwise, $a_{52}=0$ would imply either that $c_{5}=0$, and also that stages 1 and 5 are equivalent, or else that $c_{5}=$ $c_{4}$, and that stages 4 and 5 are equivalent. Since both alternatives contradict 5-minimality, it follows that $A \mathbf{q}^{2}$ and $C A \mathbf{q}^{2}$ are linearly independent. Thus, $\left\{\mathbf{q}^{2}, A \mathbf{q}^{2}, C A \mathbf{q}^{2}\right\}$ is a basis for the column space of $\Psi_{5}^{\prime}$. Hence, (6) and (7) together with $\left(1^{\prime}\right)$ are necessary for this type of method to have order 5 . Since the SOV shows that $(5)$ holds for $K_{1}=K_{2}=0$ when $c_{2}=2 c_{3} / 3$, the treatment at the beginning of this section shows that the assumptions are sufficient, and the result follows.

Because these methods are characterized by (5)-(7) with $c_{2}=2 c_{3} / 3$, and $\left(1^{\prime}\right)$, it follows that they form a subset of the family found by Owren and Zennaro [6]. For those methods with $p_{i}>3$ for some values of $i=4,5,6,7$, additional constraints must be imposed on the nodes or coefficients. Two cases 
of interest obtained by appropriate computation identify continuous methods with stage-reuse.

Theorem 2. (a) There exists a FSAL family of 5-optimal CERK methods with the $\operatorname{SOV}=(5,1,3,3,3,3,3,5)$ in the parameters $c_{2}, c_{6}, c_{7}$ and $a_{54}$.

(b) There exist two FSAL families of 5-optimal CERK methods with the $\mathrm{SOV}=(5,1,3,3,4,4,4,5)$ in the single parameter $c_{2}$.

Proof. (a) This type is characterized by Owren and Zennaro [6], and also at the beginning of this section, so $2 c_{3}=c_{4}=c_{5}$. Furthermore, $p_{3}=3$ requires the choice $c_{2}=2 c_{3} / 3$.

(b) Since $q_{3}^{4}=-9 c_{2}^{4} / 64 \neq 0$, and $c_{4}=3 c_{2}$ implies that $q_{4}^{4}=-9 c_{2}^{4} / 2 \neq 0$, it follows that $p_{3}, p_{4} \leq 3$. In order to have $p_{5}=4, a_{54}$ must be constrained. To have $p_{6}=4$, one must have $c_{6}=5 c_{2} / 3$. To have $p_{7}=4, c_{7}$ must be the zero of a quartic. Two values, $c_{7}=0$ and $c_{7}=c_{6}$ lead to equivalent stages, each of which violates 5-minimality. The other two values lead to methods that have real coefficients only if

$1822500 c_{2}^{6}-2430000 c_{2}^{5}+1291500 c_{2}^{4}-322200 c_{2}^{3}+35625 c_{2}^{2}-1320 c_{2}+16 \geq 0$.

Values of $c_{2}$ in $[0,1]$ that satisfy (29) lie outside a subinterval approximately equal to [.21536,.27767]. Some, but not all, choices will lead to methods for which all nodes lie in the interval $[0,1]$.

Other combinations of values of $p_{i}, i=5,6,7$, are possible. However, Theorem 2 identifies the extreme choices, and none lead to a method with DSO $=4$. This completes the classification of 5-optimal CERK methods.

\section{SOME METHODS}

We conclude the paper with examples of several methods. Only the discrete coefficients are given. Weights for the final stages of orders 4 and 5 are identified as $\mathbf{b}^{4}$ and $\mathbf{b}^{5}$, respectively. For each FSAL method, stage 8 is the same as the propagating stage of order 5 , so that these coefficients are not repeated. For each method in Tables 2-6, which have DSO $=1$, the eight weight functions $\left\{b_{i}(u), i=1, \ldots, 8\right\}$ for the interpolant may be obtained by solving the quadrature conditions $\left(1^{\prime}\right)$ and the three constraints $\mathbf{b}^{t}(u) \mathbf{q}^{2}=\mathbf{b}^{t}(u) C \mathbf{q}^{2}=$ $\mathbf{b}^{t}(u) C^{2} \mathbf{q}^{2}=0$. Otherwise, for methods in Tables $7-9$, for which DSO $\geq 2$, the weight functions are obtained by solving the quadrature conditions and the three constraints $\left(\mathbf{b}^{t}(u)\right)_{2}=\left(\mathbf{b}^{t}(u) A\right)_{2}=\left(\mathbf{b}^{t}(u) C A\right)_{2}=0$.

Arbitrary nodes were selected in order to illustrate structural differences between the various types of methods. Thus, it will be observed that for each pair of methods, that many (and for the first three methods all) of the nodes are selected to be equal. Otherwise, the nodes were selected so that the coefficients could be represented exactly with a small number of digits. It is quite likely that more efficient methods can be obtained by other choices of the nodes (see [6]). 
TABLE 2. A CERK(8:5) method with $\operatorname{SOV}=(5,1,1,1,4,1,1,4)$

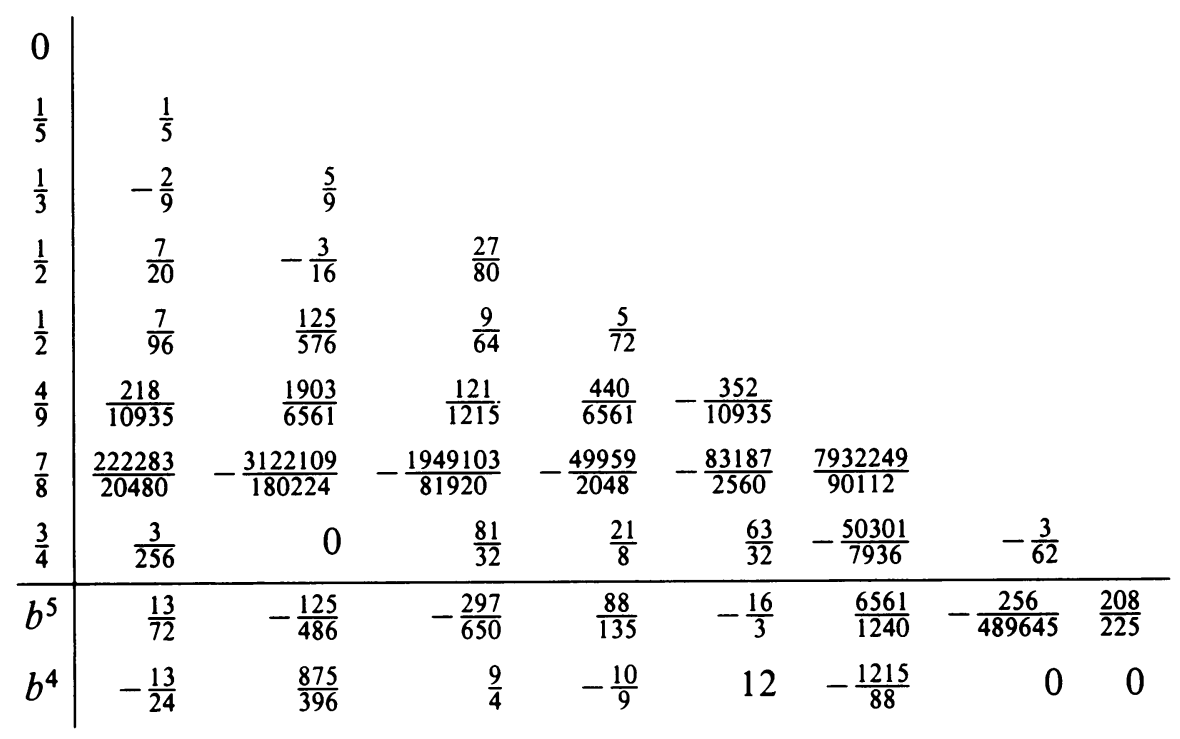

TABLE 3. $A \operatorname{CERK}(8: 5)$ method with $\operatorname{SOV}=(5,1,1,1,4,1,4,1)$

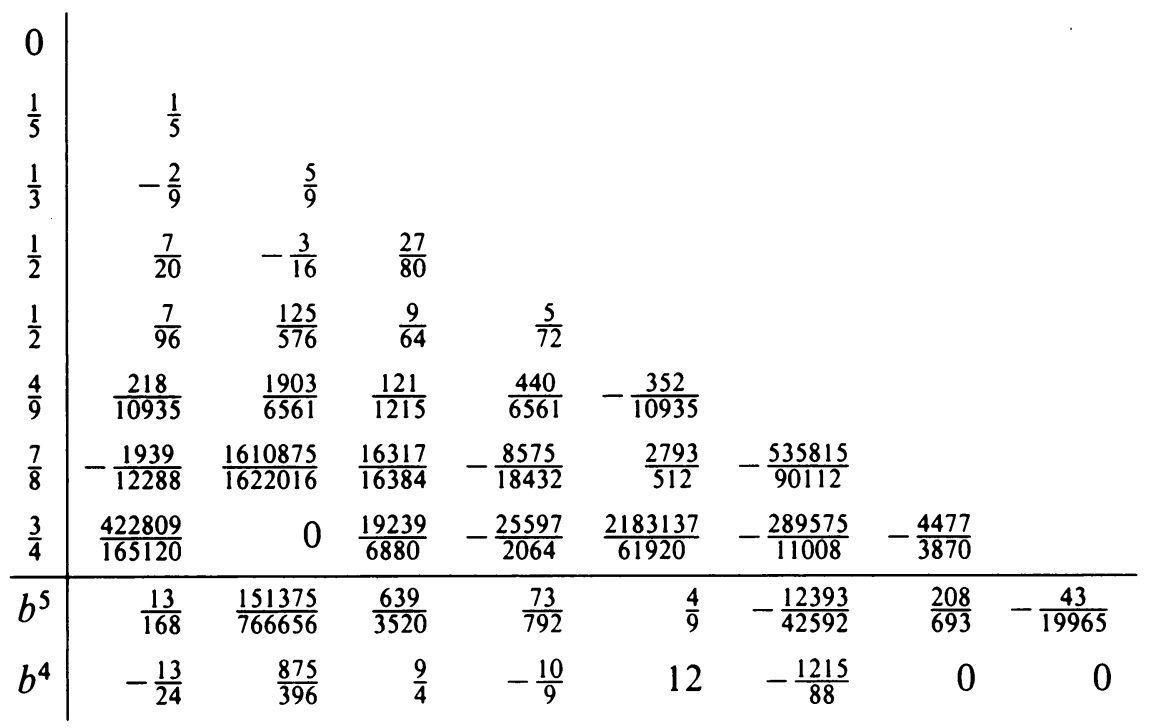


TABLE 4. A CERK(8:5) method with $\operatorname{SOV}=(5,1,1,1,4,1,4,4)$

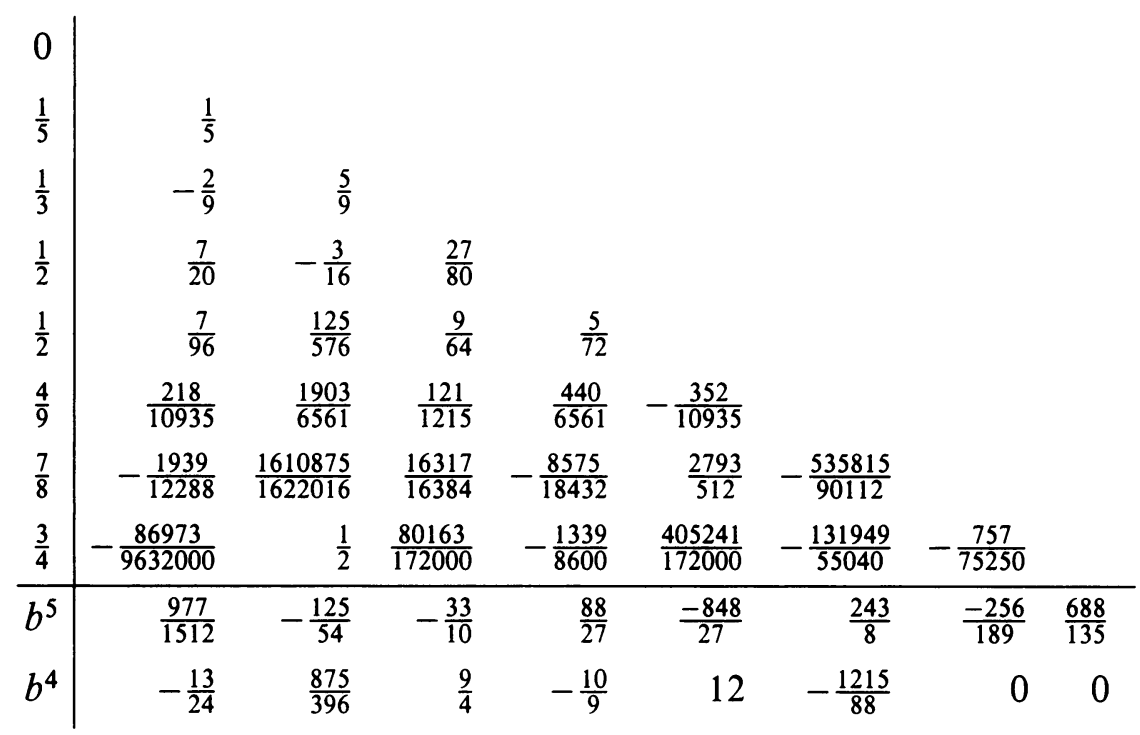

TABLE 5. A FSAL CERK(8:5) method with $\operatorname{SOV}=(5,1,1,1,4,1,1,5)$

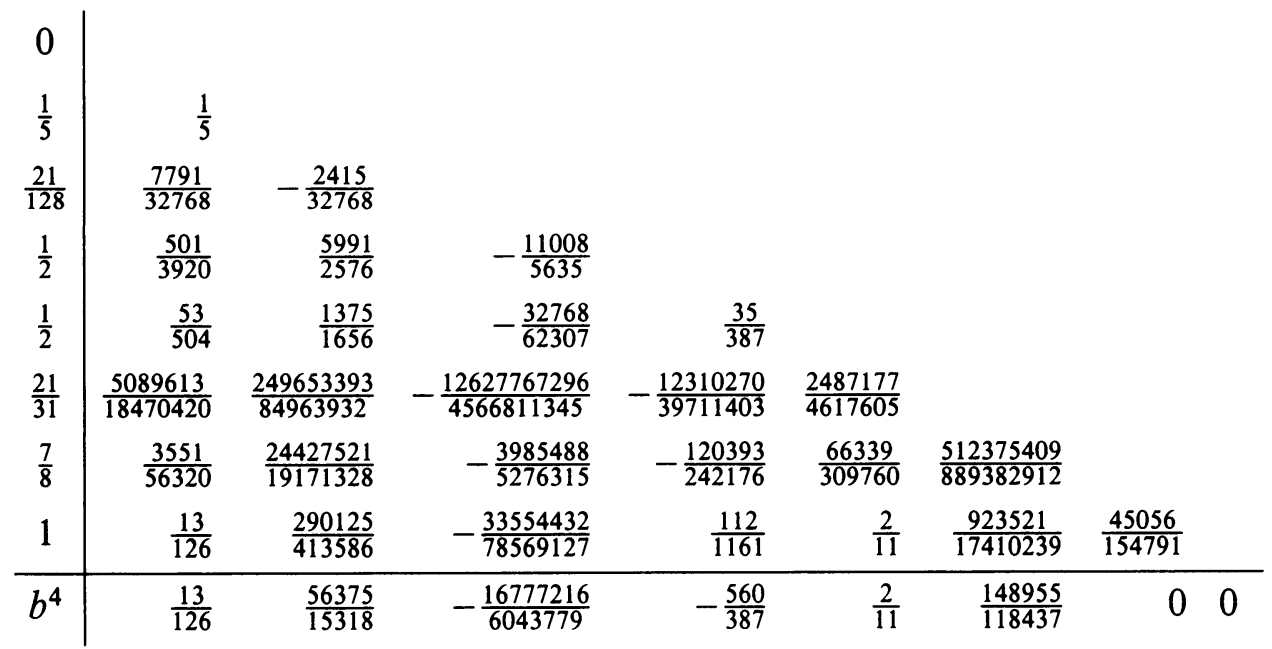


TABLE 6. A FSAL CERK(8:5) method with $\operatorname{SOV}=(5,1,1,1,4,1,4,5)$

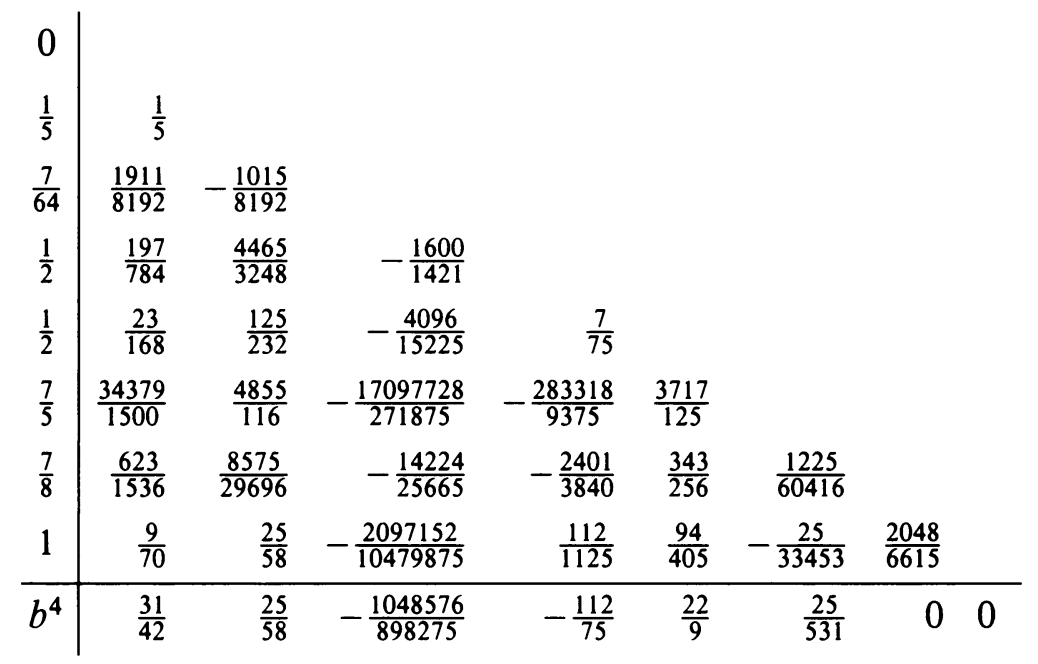

TABLE 7. A FSAL CERK(8:5) method with $\operatorname{SOV}=(5,1,2,2,2,2,2,5)$

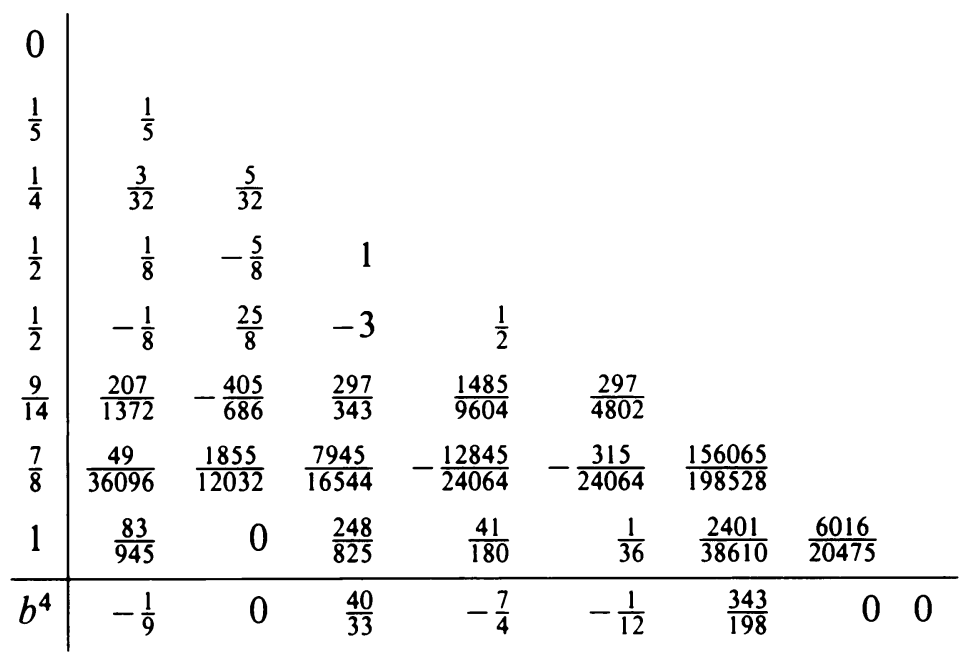


TABLE 8. A FSAL CERK(8:5) method with $\operatorname{SOV}=(5,1,3,3,3,3,3,5)$

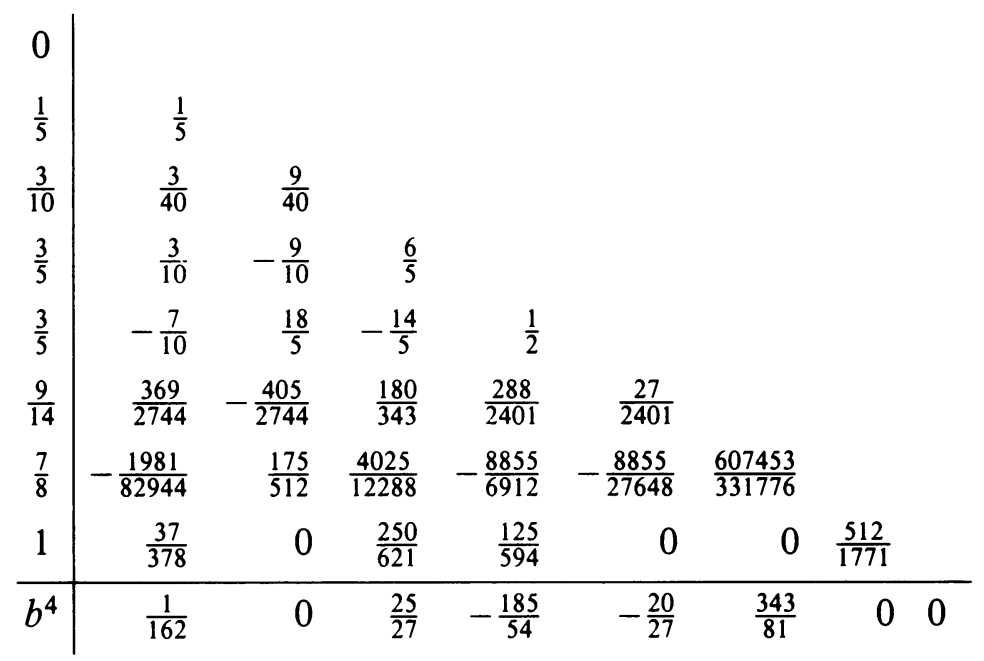

TABLE 9. A FSAL CERK(8:5) method with $\operatorname{SOV}=(5,1,3,3,4,4,4,5)$

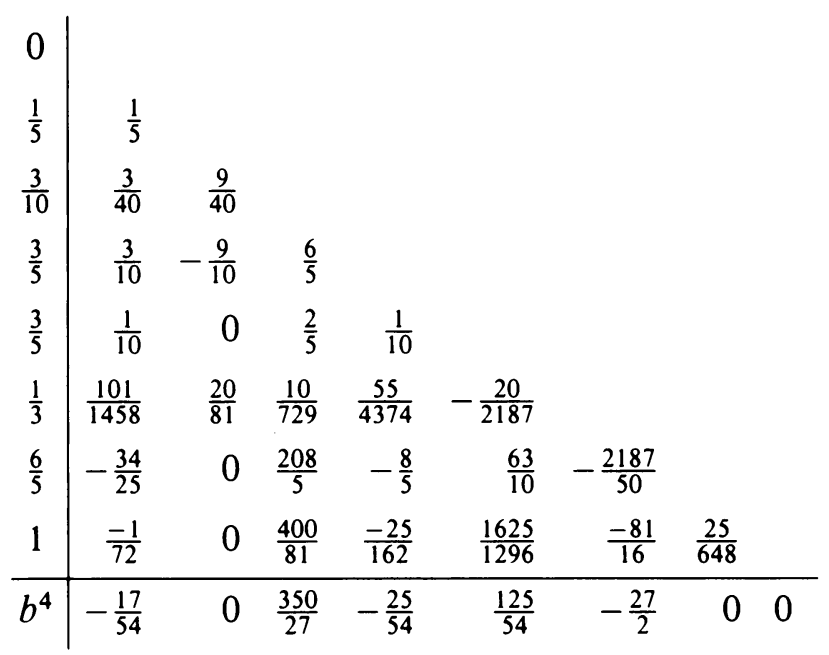

\section{ACKNOWLEDGMENTS}

This research was started in 1992, when the first author was a C.N.R. visitor at the Università degli Studi de L'Aquila, Italy. Collaboration continued in 1993 at the Department of Mathematics and Statistics of the University of Auckland, New Zealand, and the authors thank J.C. Butcher, whose contribution helped complete this work. 


\section{BIBLIOGRAPHY}

1. J. C. Butcher, The numerical analysis of ordinary differential equations, Wiley, Chichester, 1987.

2. J.R. Dormand and P.J. Prince, A family of embedded Runge-Kutta formulae, J. Comput. Appl. Math. 6 (1980), 19-26.

3. W.H. Enright, K.R. Jackson, S.P. Nørsett, and P.G. Thomsen, Interpolants for Runge-Kutta formulas, ACM Trans. Math. Software 12 (1986), 193-218.

4. E. Fehlberg, Klassische Runge-Kutta-Formeln fünfter und siebenter Ordnung mit Schrittweiten-Kontrolle, Computing 4 (1969), 93-106.

5. B. Owren and M. Zennaro, Order barriers for continuous explicit Runge-Kutta methods, Math. Comp. 56 (1991), 645-661.

6. _ Derivation of efficient continuous explicit Runge-Kutta methods, SIAM J. Sci. Statist. Comput. 13 (1992), 1488-1501.

7. P.J. Prince and J.R. Dormand, High order embedded Runge-Kutta formulae, J. Comput. Appl. Math. 7 (1981), 67-76.

8. M. Santo, Metodi continui ad un passo per la risoluzione numerica di equazioni differenziali ordinarie, Ph.D. thesis, Univ. of Udine, Italy, 1991.

9. J.H. Verner, Explicit Runge-Kutta methods with estimates of the local truncation error, SIAM J. Numer. Anal. 15 (1978), 772-790.

10. _ Differentiable interpolants for high-order Runge-Kutta methods, SIAM J. Numer. Anal. 30 (1993), 1446-1466.

11. J.H. Verner and M. Zennaro, Continuous explicit Runge-Kutta methods of order 5, Report 1993-08, Department of Mathematics and Statistics, Queen's University, Kingston, Ontario, Canada (1993), 1-32.

Department of Mathematics and Statistics, Queen's University, Kingston, Ontario, CANADA K7L 3N6

E-mail address: jim@jhv.mast.queensu.ca

Dipartimento di Scienze Matematiche, Università degli Studi di Trieste, 34100 TriESTE, ITALY

E-mail address: zennaro@univ.trieste.it 